\title{
Adjoint and Frobenius Pairs of Functors for Corings
}

\author{
M. Zarouali-Darkaoui \\ Departamento de Álgebra \\ Facultad de Ciencias \\ Universidad de Granada \\ E18071 Granada, Spain \\ E-mail: zaroual@correo.ugr.es
}

\section{Introduction}

Corings were introduced by M. Sweedler in [34 as a generalization of coalgebras over commutative rings to the case of non-commutative rings, to give a formulation of a predual of the Jacobson-Bourbaki's theorem for intermediate extensions of division ring extensions. Thus, a coring over an associative ring with unit $A$ is a comonoid in the monoidal category of all $A$-bimodules. Recently, motivated by an observation of M. Takeuchi, namely that an entwining structure (resp. an entwined module) can be viewed as a suitable coring (resp. as a comodule over a suitable coring), T. Brzeziński, has given in [5] some new examples and general properties of corings. Among them, a study of Frobenius corings is developed, extending previous results on entwining structures [4] and relative Hopf modules [11].

A pair of functors $(F, G)$ is said to be a Frobenius pair [13] if $G$ is at the same time a left and right adjoint to $F$. That is a standard name which we use instead of Morita's original "strongly adjoint pairs" 27. The functors $F$ and $G$ are known as Frobenius functors [11. The study of Frobenius functors was motivated by a paper of K. Morita, where he proved [27. Theorem 5.1] that given a ring extension $i: A \rightarrow B$, the induction functor $-\otimes_{A} B: \mathcal{M}_{A} \rightarrow \mathcal{M}_{B}$ is a Frobenius functor if and only if the morphism $i$ is Frobenius in the sense of [22] (see also [28]). The dual result for coalgebras over fields was proved in [13, Theorem 3.5] and it states that the co-restriction functor $(-)_{\varphi}: \mathcal{M}^{C} \rightarrow \mathcal{M}^{D}$ associated to a morphism of coalgebras $\varphi: C \rightarrow D$ is Frobenius if and only if $C_{D}$ is quasifinite and injective and there exists an isomorphism of bicomodules $\mathrm{h}_{D}\left({ }_{C} C_{D}, D\right) \cong{ }_{D} C_{C}$ (here, $\mathrm{h}_{D}(C,-)$ denotes the "cohom" functor). Since corings generalize both rings and coalgebras over fields, it is natural to guess that [27, Theorem 5.1] and [13, Theorem 3.5] are specializations of a general statement on homomorphisms of corings. In this paper, we find such a result (Theorem 4.1), and we introduce the notion of a (right) Frobenius extension of corings (see Definition 4.2). To prove such a result, we study adjoint pairs and Frobenius pairs of functors between categories of comodules over rather general corings. Precedents for categories of modules, and categories of comodules over coalgebras over 
fields are contained in [27], [36, [13] and [9]. More recently, Frobenius corings (i.e., corings for which the functor forgetting the coaction is Frobenius), have been intensively studied in [5, 6, 7, 8, 9].

We think that our general approach produces results of independent interest, beyond the aforementioned extension to the coring setting of [27, Theorem 5.1] and 13, Theorem 3.5], and contributes to the understanding of the subtle behavior of the cotensor product functor for corings. In fact, our general results, although sometimes rather technical, have other applications and will probably find more. For instance, the author has used them to prove new results on equivalences of comodule categories over corings [?]. Moreover, when applied to corings stemming from different algebraic theories of current interest, they boil down to new (more concrete) results. As an illustration, we consider entwined modules over an entwining structure in Section 5 , and graded modules over $G$-sets in Section 6 ,

The paper is organized as follows. After Section 1, devoted to fix some basic notations, Section 2 deals with adjoint and Frobenius pairs on categories of comodules. Some refinements of results from [18] and [8, §23] on the representation as cotensor product functors of certain functors between comodule categories are needed and are thus included in Section 2. From our general discussion on adjoint pairs of cotensor product functors we will derive our main general result on Frobenius pairs between comodule categories (Theorem 2.10) that extends the known characterizations in the setting of modules over rings and of comodules over coalgebras. In the first case, the key property to derive the result on modules from Theorem 2.10] is the separability of the trivial corings (see Remark 2.12). In the case of coalgebras, the fundamental additional property is the duality between finite left and right comodules. We already consider a much more general situation in Section 3. where we introduce the class of so called corings having a duality for which we prove characterizations of Frobenius pairs that are similar to the coalgebra case.

Section 4 is the leitmotiv of the paper. After the technical development of sections 2 and 3. our main results follow without difficulty. We prove in particular that the induction functor $-\otimes_{A} B: \mathcal{M}^{\mathfrak{C}} \rightarrow \mathcal{M}^{\mathfrak{D}}$ associated to a homomorphism $(\varphi, \rho): \mathfrak{C} \rightarrow \mathfrak{D}$ of corings $\mathfrak{C}$ and $\mathfrak{D}$ flat over their respective base rings $A$ and $B$ is Frobenius if and only if the $\mathfrak{C}-\mathfrak{D}$ bicomodule $\mathfrak{C} \otimes_{A} B$ is quasi-finite and injector as a right $\mathfrak{D}$-comodule and there exists an isomorphism of $\mathfrak{D}-\mathfrak{C}$-bicomodules $\mathrm{h}_{\mathfrak{D}}\left(\mathfrak{C} \otimes_{A} B, \mathfrak{D}\right) \simeq B \otimes_{A} \mathfrak{C}$ (Theorem 4.1). We show as well how this theorem unifies previous results for ring homomorphisms [27, Theorem 5.1], coalgebra maps [13, Theorem 3.5], and Frobenius corings [8, 27.10, 28.8].

In Section 5 , we specialize one of the general results on corings to entwining structures.

In Section [6] we particularize our results in the previous sections to the coring associated to a $G$-graded algebra and a $G$-set, where $G$ ia a group. We obtain a series of new results for graded modules by $G$-sets.

\section{Basic notations}

Throughout this paper and unless otherwise stated, $k$ denote a commutative ring (with unit), $A, A^{\prime}, A^{\prime \prime}$, and $B$ denote associative and unitary algebras over $k$, and $\mathfrak{C}, \mathfrak{C}^{\prime \prime}, \mathfrak{C}^{\prime \prime}$, and 
$\mathfrak{D}$ denote corings over $A, A^{\prime}, A^{\prime \prime}$, and $B$, respectively. We recall from 34 that an $A$-coring consists of an $A$-bimodule $\mathfrak{C}$ with two $A$-bimodule maps

$$
\Delta: \mathfrak{C} \longrightarrow \mathfrak{C} \otimes_{A} \mathfrak{C}, \quad \epsilon: \mathfrak{C} \longrightarrow A
$$

such that $\left(\mathfrak{C} \otimes_{A} \Delta\right) \circ \Delta=\left(\Delta \otimes_{A} \mathfrak{C}\right) \circ \Delta$ and $\left(\epsilon \otimes_{A} \mathfrak{C}\right) \circ \Delta=\left(\mathfrak{C} \otimes_{A} \epsilon\right) \circ \Delta=1_{\mathfrak{C}}$. A right $\mathfrak{C}$-comodule is a pair $\left(M, \rho_{M}\right)$ consisting of a right $A$-module $M$ and an $A$-linear map $\rho_{M}: M \rightarrow M \otimes_{A} \mathfrak{C}$ (the coaction) satisfying $\left(M \otimes_{A} \Delta\right) \circ \rho_{M}=\left(\rho_{M} \otimes_{A} \mathfrak{C}\right) \circ \rho_{M}$, $\left(M \otimes_{A} \epsilon\right) \circ \rho_{M}=1_{M}$. A morphism of right $\mathfrak{C}$-comodules $\left(M, \rho_{M}\right)$ and $\left(N, \rho_{N}\right)$ is a right $A$-linear map $f: M \rightarrow N$ such that $\left(f \otimes_{A} \mathfrak{C}\right) \circ \rho_{M}=\rho_{N} \circ f$; the $k$-module of all such morphisms will be denoted by $\operatorname{Hom}_{\mathfrak{C}}(M, N)$. The right $\mathfrak{C}$-comodules together with their morphisms form the additive category $\mathcal{M}^{\mathfrak{c}}$. Coproducts and cokernels (and then inductive limits) in $\mathcal{M}^{\mathfrak{C}}$ exist and they coincide respectively with coproducts and cokernels in the category of right $A$-modules $\mathcal{M}_{A}$. If ${ }_{A} \mathfrak{C}$ is flat, then $\mathcal{M}^{\mathfrak{C}}$ is a Grothendieck category. The converse is not true in general (see [16, Example 1.1]). When $\mathfrak{C}=A$ with the trivial $A$-coring structure, then $\mathcal{M}^{A}=\mathcal{M}_{A}$.

Now assume that the $A^{\prime}-A$-bimodule $M$ is also a left comodule over an $A^{\prime}$-coring $\mathfrak{C}^{\prime}$ with structure map $\lambda_{M}: M \rightarrow \mathfrak{C}^{\prime \prime} \otimes_{A^{\prime}} M$. Assume moreover that $\rho_{M}$ is $A^{\prime}$-linear, and $\lambda_{M}$ is $A$-linear. It is clear that $\rho_{M}: M \rightarrow M \otimes_{A} \mathfrak{C}$ is a morphism of left $\mathfrak{C}^{\prime \prime}$-comodules if and only if $\lambda_{M}: M \rightarrow \mathfrak{C}^{\prime} \otimes_{A^{\prime}} M$ is a morphism of right $\mathfrak{C}$-comodules. In this case, we say that $M$ is a $\mathfrak{C}^{\prime}-\mathfrak{C}$-bicomodule. The $\mathfrak{C}^{\prime}-\mathfrak{C}^{\mathfrak{C}}$-bicomodules are the objects of a category ${ }^{\mathfrak{C}^{\prime}} \mathcal{M}^{\mathfrak{C}}$, whose morphisms are defined in the obvious way.

Let $Z$ be a left $A$-module and $f: X \rightarrow Y$ a morphism in $\mathcal{M}_{A}$. Following [8, 40.13] we say that $f$ is $Z$-pure when the functor $-\otimes_{A} Z$ preserves the kernel of $f$. If $f$ is $Z$-pure for every $Z \in{ }_{A} \mathcal{M}$ then we say simply that $f$ is pure in $\mathcal{M}_{A}$. The notation $\otimes$ will stand for the tensor product over $k$.

\section{Frobenius functors between categories of comodules}

Let $T$ be a $k$-algebra, and $M \in{ }^{T} \mathcal{M}^{A}$. Let $\varphi: T \rightarrow \operatorname{End}_{A}(M)$ the morphism of $k$-algebras given by the right $T$-module structure of the bimodule ${ }_{T} M_{A}$. Now, suppose moreover that $M \in \mathcal{M}^{\mathfrak{C}}$. Then $\operatorname{End}_{\mathfrak{C}}(M)$ is a subalgebra of $\operatorname{End}_{A}(M)$. We have that $\varphi(T) \subset \operatorname{End}_{\mathfrak{C}}(M)$ if and only if $\rho_{M}$ is $T$-linear. Hence the left $T$-module structure of a $T-\mathfrak{C}$-bicomodule $M$ can be described as a morphism of $k$-algebras $\varphi: T \rightarrow \operatorname{End}_{\mathfrak{c}}(M)$. Given a $k$-linear functor $F$ : $\mathcal{M}^{\mathfrak{C}} \rightarrow \mathcal{M}^{\mathfrak{D}}$, and $M \in{ }^{T} \mathcal{M}^{\mathfrak{C}}$, the algebra morphism $T \stackrel{\varphi}{\longrightarrow} \operatorname{End}_{\mathfrak{C}}(M) \stackrel{F(-)}{\longrightarrow} \operatorname{End}_{\mathfrak{D}}(F(M))$ defines a $T-\mathfrak{D}$-bicomodule structure on $F(M)$. We have then two $k$-linear bifunctors

$$
-\otimes_{T} F(-), F\left(-\otimes_{T}-\right): \mathcal{M}^{T} \times{ }^{T} \mathcal{M}^{\mathfrak{C}} \rightarrow \mathcal{M}^{\mathfrak{D}} .
$$


Let $\Upsilon_{T, M}$ be the unique isomorphism of $\mathfrak{D}$-comodules making the following diagram commutative

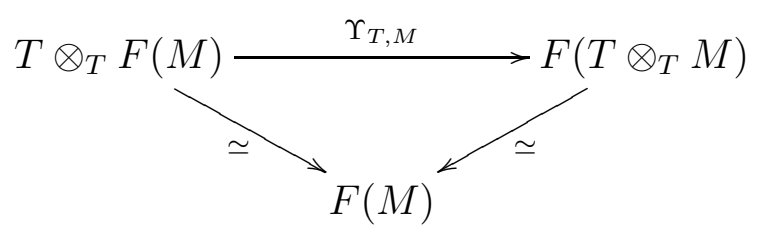

for every $M \in{ }^{T} \mathcal{M}^{\mathfrak{C}}$. We have that $\Upsilon_{T, M}$ is natural in $T$. By the theorem of Mitchell [31, Theorem 3.6.5], there exists a unique natural transformation

$$
\Upsilon_{-, M}:-\otimes_{T} F(M) \rightarrow F\left(-\otimes_{T} M\right)
$$

extending the natural transformation $\Upsilon_{T, M}$. We refer to [18] for more details.

Remark 2.1. Mitchell's [31, Theorem 3.6.5] holds also if we only suppose that the target category $\mathscr{C}^{\prime}$ is preadditive and has coproducts, or if the category $\mathscr{C}^{\prime}$ is preadditive and the functor $S$ preserves coproducts (notations as in [31, Theorem 3.6.5]). This fact is used to show that the natural transformation $\Upsilon$ exists for every $k$-linear functor $F: \mathcal{M}^{\mathfrak{C}} \rightarrow \mathcal{M}^{\mathfrak{D}}$ even if the category $\mathcal{M}^{\mathfrak{D}}$ is not abelian. Note also that its corollary [31, Corollary 3.6.6] also holds if we suppose only that the category $\mathscr{C}^{\prime}$ is preadditive.

Let $M \in \mathfrak{C}^{\mathfrak{C}^{\prime}} \mathcal{M}^{\mathfrak{C}}$ and $N \in \mathfrak{C}^{\mathfrak{C}} \mathcal{M}^{\mathfrak{C}^{\prime \prime}}$. The map

$$
\omega_{M, N}=\rho_{M} \otimes_{A} N-M \otimes_{A} \lambda_{N}: M \otimes_{A} N \rightarrow M \otimes_{A} \mathfrak{C} \otimes_{A} N
$$

is a $\mathfrak{C}^{\prime}-\mathfrak{C}^{\prime \prime}$-bicomodule map. Its kernel in ${ }_{A^{\prime}} \mathcal{M}_{A^{\prime \prime}}$ is the cotensor product of $M$ and $N$, and it is denoted by $M \square_{\mathfrak{C}} N$. If $\omega_{M, N}$ is $\mathfrak{C}_{A^{\prime}}^{\prime \prime}$-pure and ${ }_{A^{\prime \prime}} \mathfrak{C}^{\mathfrak{C}^{\prime \prime}}$-pure, and the following

$$
\operatorname{ker}\left(\omega_{M, N}\right) \otimes_{A^{\prime \prime}} \mathfrak{C}^{\prime \prime} \otimes_{A^{\prime \prime}} \mathfrak{C}^{\prime \prime}, \quad \mathfrak{C}^{\prime} \otimes_{A^{\prime}} \mathfrak{C}^{\prime} \otimes_{A^{\prime}} \operatorname{ker}\left(\omega_{M, N}\right) \quad \text { and } \quad \mathfrak{C}^{\prime} \otimes_{A^{\prime}} \operatorname{ker}\left(\omega_{M, N}\right) \otimes_{A^{\prime \prime}} \mathfrak{C}^{\prime \prime}
$$

are injective maps, then $M \square_{\mathfrak{C}} N$ is the kernel of $\omega_{M, N}$ in ${ }^{\mathfrak{C}^{\prime}} \mathcal{M}^{\mathfrak{C}^{\prime \prime}}$. This is the case if $\omega_{M, N}$ is $\left(\mathfrak{C}^{\prime} \otimes_{A^{\prime}} \mathfrak{C}^{\prime \prime}\right)_{A^{\prime}}$-pure, $A_{A^{\prime \prime}}\left(\mathfrak{C}^{\prime \prime \prime} \otimes_{A^{\prime \prime}} \mathfrak{C}^{\prime \prime \prime}\right)$-pure, and $\mathfrak{C}^{\prime \prime} \otimes_{A^{\prime}} \omega_{M, N}$ is $A_{A^{\prime \prime}} \mathfrak{C}^{\prime \prime \prime}$-pure (e.g. if $\mathfrak{C}_{A^{\prime}}^{\prime}$ and ${ }_{A^{\prime \prime}} \mathfrak{C}^{\prime \prime \prime}$ are flat, or if $\mathfrak{C}$ is a coseparable $A$-coring).

If for every $M \in \mathfrak{C}^{\mathfrak{C}^{\prime}} \mathcal{M}^{\mathfrak{C}}$ and $N \in{ }^{\mathfrak{C}} \mathcal{M}^{\mathfrak{C}^{\prime \prime}}, \omega_{M, N}$ is $\mathfrak{C}_{A^{\prime}}^{\prime \prime}$-pure and $A^{\prime \prime} \mathfrak{C}^{\prime \prime \prime}$-pure, then we have a $k$-linear bifunctor

$$
-\square_{\mathfrak{C}}-: \mathfrak{C}^{\mathfrak{C}^{\prime}} \mathcal{M}^{\mathfrak{C}} \times{ }^{\mathfrak{c}} \mathcal{M}^{\mathfrak{C}^{\prime \prime}} \longrightarrow \mathfrak{C}^{\mathfrak{C}^{\prime}} \mathcal{M}^{\mathfrak{C}^{\prime \prime}}
$$

If in particular $\mathfrak{C}_{A^{\prime}}^{\prime}$ and ${ }_{A^{\prime \prime}} \mathfrak{C}^{\prime \prime}$ are flat, or if $\mathfrak{C}$ is a coseparable $A$-coring, then the bifunctor (3) is well defined.

By a proof similar to that of [1, II.1.3], we have, for every $M \in \mathcal{M}^{\mathfrak{C}}$, that the functor $M \square_{\mathfrak{C}}-$ preserves direct limits.

The following lemma was used implicitly in the proof of [18, Proposition 3.4], and it will be useful for us in the proof of the next theorem. 
Lemma 2.2. If $M \in \mathfrak{C}^{\mathfrak{C}^{\prime}} \mathcal{M}^{\mathfrak{C}}$, and $F: \mathcal{M}^{\mathfrak{C}} \rightarrow \mathcal{M}^{\mathfrak{D}}$ is a $M$-compatible $k$-linear functor in the sense of [18, $p$. 210], which preserves coproducts, then for all $X \in \mathcal{M}^{A^{\prime}}$,

$$
\Upsilon_{X \otimes_{A^{\prime}} \mathfrak{C}^{\prime}, M}\left(X \otimes_{A^{\prime}} \lambda_{F(M)}\right)=F\left(X \otimes_{A^{\prime}} \lambda_{M}\right) \Upsilon_{X, M}
$$

Proof. Let us consider the diagram

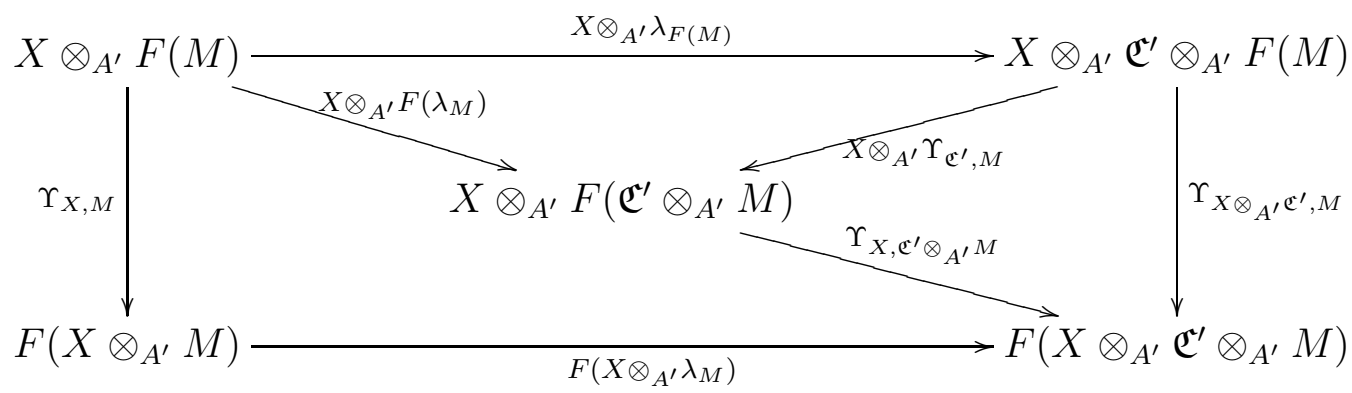

The commutativity of the top triangle follows from the definition of $\lambda_{F(M)}$, while the right triangle commutes by [18, Lemma 3.3] (we take $S=T=A^{\prime}$, and $Y=\mathfrak{C}^{\prime}$ ), and the left triangle is commutative since $\Upsilon_{X,-}$ is natural. Therefore, the commutativity of the rectangle holds.

A closer analysis of [18, Theorem 3.5] gives the following generalization of [36, Proposition 2.1] and [8, 23.1(1)]. Recall from [21] that a coring $\mathfrak{C}$ is said to be coseparable if the comultiplication map $\Delta_{\mathfrak{C}}$ is a split monomorphism of $\mathfrak{C}$-bicomodules. Of course, the trivial $A$-coring $\mathfrak{C}=A$ is coseparable and, henceforth, every result for comodules over coseparable corings applies in particular for modules over rings.

Theorem 2.3. Let $F: \mathcal{M}^{\mathfrak{C}} \rightarrow \mathcal{M}^{\mathfrak{D}}$ be a $k$-linear functor, such that

(I) ${ }_{B} \mathfrak{D}$ is flat and $F$ preserves the kernel of $\rho_{N} \otimes_{A} \mathfrak{C}-N \otimes_{A} \Delta_{\mathfrak{C}}$ for every $N \in \mathcal{M}^{\mathfrak{C}}$, or

(II) $\mathfrak{C}$ is a coseparable $A$-coring and the categories $\mathcal{M}^{\mathfrak{C}}$ and $\mathcal{M}^{\mathfrak{D}}$ are abelian.

Assume that at least one of the following statements holds

1. $\mathfrak{C}_{A}$ is projective, $F$ preserves coproducts, and $\Upsilon_{N, \mathfrak{C}}, \Upsilon_{N \otimes_{A} \mathfrak{C}, \mathfrak{C}}$ are isomorphisms for all $N \in \mathcal{M}^{\mathfrak{C}}$ (e.g. , if $A$ is semisimple and $F$ preserves coproducts), or

2. $\mathfrak{C}_{A}$ is flat, $F$ preserves direct limits, and $\Upsilon_{N, \mathfrak{C}}, \Upsilon_{N \otimes_{A} \mathfrak{C}, \mathfrak{C}}$ are isomorphisms for all $N \in \mathcal{M}^{\mathfrak{C}}$ (e.g. , if $A$ is a von Neumann regular ring and $F$ preserves direct limits), or

3. F preserves inductive limits (e.g., if F has a right adjoint).

Then $F$ is naturally equivalent to $-\square_{\mathfrak{C}} F(\mathfrak{C})$. 
Proof. At first, note that if $\mathfrak{C}_{A}$ is projective, then the right $A$-module $\mathfrak{C} \otimes_{A} \mathfrak{C}$ is projective (by [33, Example 3 p. 105, Proposition VI.9.5]). Hence, if $\mathfrak{C}_{A}$ is projective and $F$ preserves coproducts, then $F$ is $M$-compatible in the sense of [18, p. 210], for all $M \in{ }^{\mathfrak{C}} \mathcal{M}^{\mathfrak{C}}$. In each case, we have $F$ is $\mathfrak{C}$-compatible where $\mathfrak{C} \in{ }^{\mathfrak{C}} \mathcal{M}^{\mathfrak{C}}$. Therefore, by [18, Proposition 3.4], $F(\mathfrak{C})$ can be viewed as a $\mathfrak{C}-\mathfrak{D}$-bicomodule. From Lemma 2.2 and since $\Upsilon_{-, \mathfrak{C}}$ is a natural transformation, we have, for every $N \in \mathcal{M}^{\mathfrak{C}}$, the commutativity of the following diagram with exact rows in $\mathcal{M}^{\mathfrak{D}}$

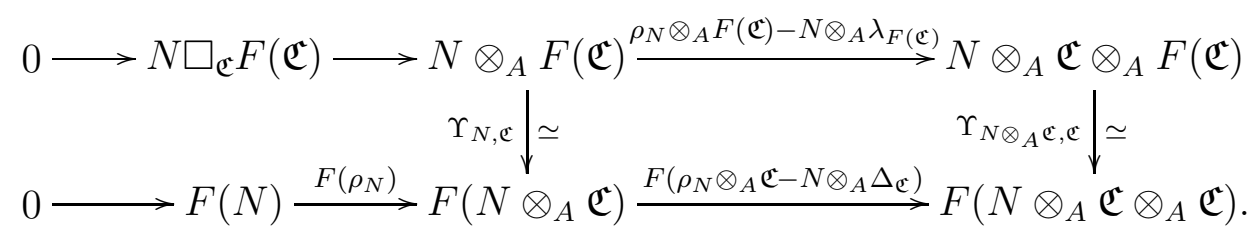

The exactness of the bottom sequence is assumed in the case $(I)$. For the case $(I I)$, it follows by factorizing the map $\omega_{N, \mathfrak{C}}=\rho_{N} \otimes_{A} \mathfrak{C}-N \otimes_{A} \Delta_{\mathfrak{C}}$ through its image, and using the facts that the sequence $0 \longrightarrow N \stackrel{\rho_{N}}{\longrightarrow} N \otimes_{A} \mathfrak{C} \stackrel{\omega_{N, \mathfrak{c}}}{\longrightarrow} N \otimes_{A} \mathfrak{C} \otimes_{A} \mathfrak{C}$ is split exact in $\mathcal{M}^{\mathfrak{C}}$ in the sense of [8, 40.5], and that additive functors between abelian categories preserve split exactness. By the universal property of kernels, there exists a unique isomorphism $\eta_{N}: N \square_{\mathfrak{C}} F(\mathfrak{C}) \rightarrow F(N)$ in $\mathcal{M}^{\mathfrak{D}}$ making commutative the above diagram. It easy to show that $\eta$ is natural. Hence $F \simeq-\square_{\mathfrak{C}} F(\mathfrak{C})$.

As an immediate consequence of the last theorem we have the following generalization of Eilenberg-Watts Theorem [33, Proposition VI.10.1].

Corollary 2.4. Let $F: \mathcal{M}^{\mathfrak{C}} \rightarrow \mathcal{M}^{\mathfrak{D}}$ be a $k$-linear functor.

1. If ${ }_{B} \mathfrak{D}$ is flat and $A$ is a semisimple ring (resp. a von Neumann regular ring), then the following statements are equivalent

(a) F is left exact and preserves coproducts (resp. left exact and preserves direct limits);

(b) $F \simeq-\square_{\mathfrak{C}} M$ for some bicomodule $M \in{ }^{\mathfrak{C}} \mathcal{M}^{\mathfrak{D}}$.

2. If ${ }_{A} \mathfrak{C}$ and ${ }_{B} \mathfrak{D}$ are flat, then the following statements are equivalent

(a) $F$ is exact and preserves inductive limits;

(b) $F \simeq-\square_{\mathfrak{C}} M$ for some bicomodule $M \in{ }^{\mathfrak{C}} \mathcal{M}^{\mathfrak{D}}$ which is coflat in ${ }^{\mathfrak{c}} \mathcal{M}$.

3. If $\mathfrak{C}$ is a coseparable A-coring and the categories $\mathcal{M}^{\mathfrak{C}}$ and $\mathcal{M}^{\mathfrak{D}}$ are abelian, then the following statements are equivalent

(a) F preserves inductive limits;

(b) $F$ preserves cokernels and $F \simeq-\square_{\mathfrak{C}} M$ for some bicomodule $M \in{ }^{\mathfrak{C}} \mathcal{M}^{\mathfrak{D}}$.

4. If $\mathfrak{C}=A$ and the category $\mathcal{M}^{\mathfrak{D}}$ is abelian, then the following statements are equivalent 
(a) F has a right adjoint;

(b) F preserves inductive limits;

(c) $F \simeq-\otimes_{A} M$ for some bicomodule $M \in{ }^{A} \mathcal{M}^{\mathcal{D}}$.

A bicomodule $N \in \mathfrak{C}^{\mathfrak{C}} \mathcal{M}^{\mathfrak{D}}$ is said to be quasi-finite as a right $\mathfrak{D}$-comodule if the functor $-\otimes_{A} N: \mathcal{M}_{A} \rightarrow \mathcal{M}^{\mathfrak{D}}$ has a left adjoint $\mathrm{h}_{\mathfrak{D}}(N,-): \mathcal{M}^{\mathcal{D}} \rightarrow \mathcal{M}_{A}$, the cohom functor. If $\omega_{Y, N}$ is $\mathfrak{D} \otimes_{B} \mathfrak{D}$-pure for every right $\mathfrak{C}$-comodule $Y$ (e.g., ${ }_{B} \mathfrak{D}$ is flat or $\mathfrak{C}$ is coseparable) then $N_{\mathfrak{D}}$ is quasi-finite if and only if $-\square_{\mathfrak{C}} N: \mathcal{M}^{\mathfrak{C}} \rightarrow \mathcal{M}^{\mathfrak{D}}$ has a left adjoint, which we still to denote by $\mathrm{h}_{\mathfrak{D}}(N,-)$ [18, Proposition 4.2]. The particular case of the following statement when the cohom is exact generalizes [2, Corollary 3.12].

Corollary 2.5. Let $N \in{ }^{\mathfrak{C}} \mathcal{M}^{\mathfrak{D}}$ be a bicomodule, quasi-finite as a right $\mathfrak{D}$-comodule, such that ${ }_{A} \mathfrak{C}$ and ${ }_{B} \mathfrak{D}$ are flat. If the cohom functor $\mathrm{h}_{\mathfrak{D}}(N,-)$ is exact or if $\mathfrak{D}$ is a coseparable $B$-coring, then we have

$$
\mathrm{h}_{\mathfrak{D}}(N,-) \simeq-\square_{\mathfrak{D}} \mathrm{h}_{\mathfrak{D}}(N, \mathfrak{D}): \mathcal{M}^{\mathfrak{D}} \rightarrow \mathcal{M}^{\mathfrak{C}} .
$$

Proof. The functor $\mathrm{h}_{\mathfrak{D}}(N,-)$ is $k$-linear and preserves inductive limits, since it is a left adjoint to the $k$-linear functor $-\square_{\mathfrak{C}} N: \mathcal{M}^{\mathfrak{C}} \rightarrow \mathcal{M}^{\mathfrak{D}}$ (by [18, Proposition 4.2]). Hence Theorem 2.3 achieves the proof.

Now we will use the following generalization of [36, Lemma 2.2].

Lemma 2.6. Let $\Lambda, \Lambda^{\prime}$ be bicomodules in ${ }^{\mathfrak{D}} \mathcal{M}^{\mathfrak{C}}$ and $G=-\square_{\mathfrak{D}} \Lambda, G^{\prime}=-\square_{\mathfrak{D}} \Lambda^{\prime}$. Suppose moreover that ${ }_{A} \mathfrak{C}$ is flat and $B$ is a von Neumann regular ring, or ${ }_{A} \mathfrak{C}$ is flat and $G$ and $G^{\prime}$ are cokernel preserving, or $\mathfrak{D}$ is a coseparable coring. Then

$$
\operatorname{Nat}\left(G, G^{\prime}\right) \simeq \operatorname{Hom}_{(\mathfrak{D}, \mathfrak{C})}\left(\Lambda, \Lambda^{\prime}\right) .
$$

Proof. Let $\alpha: G \rightarrow G^{\prime}$ be a natural transformation. By [18, Lemma 3.2(1)], $\alpha_{\mathfrak{D}}$ is left $B$-linear. For the rest of the proof it suffices to replace $\otimes$ by $\otimes_{B}$ in the proof of [9. Lemma 4.1].

The following proposition generalizes [27, Theorem 2.1] from bimodules over rings to bicomodules over corings.

Proposition 2.7. Suppose that ${ }_{A} \mathfrak{C}, \mathfrak{C}_{A},{ }_{B} \mathfrak{D}$ and $\mathfrak{D}_{B}$ are flat. Let $X \in \mathfrak{C}^{\mathfrak{D}} \mathcal{D}^{\text {and }} \Lambda \in$ $\mathfrak{D}_{\mathcal{M}}^{\mathfrak{C}}$. Consider the following properties:

(1) $-\square_{\mathfrak{C}} X$ is left adjoint to $-\square_{\mathfrak{D}} \Lambda$;

(2) $\Lambda$ is quasi-finite as a right $\mathfrak{C}$-comodule and $-\square_{\mathfrak{C}} X \simeq \mathrm{h}_{\mathfrak{C}}(\Lambda,-)$;

(3) $\Lambda$ is quasi-finite as a right $\mathfrak{C}$-comodule and $X \simeq \mathrm{h}_{\mathfrak{C}}(\Lambda, \mathfrak{C})$ in ${ }^{\mathfrak{C}} \mathcal{M}^{\mathfrak{D}}$; 
(4) there exist bicolinear maps

$$
\psi: \mathfrak{C} \rightarrow X \square_{\mathfrak{D}} \Lambda \text { and } \omega: \Lambda \square_{\mathfrak{C}} X \rightarrow \mathfrak{D}
$$

in ${ }^{\mathfrak{C}} \mathcal{M}^{\mathfrak{C}}$ and ${ }^{\mathfrak{D}} \mathcal{M}^{\mathfrak{D}}$ respectively, such that

$$
\left(\omega \square_{\mathfrak{D}} \Lambda\right) \circ\left(\Lambda \square_{\mathfrak{C}} \psi\right)=\Lambda \text { and }\left(X \square_{\mathfrak{D}} \omega\right) \circ\left(\psi \square_{\mathfrak{C}} X\right)=X
$$

(5) $\Lambda \square_{\mathfrak{C}}-$ is left adjoint to $X \square_{\mathfrak{D}}-$.

Then (11) and (2) are equivalent, and they imply (3). The converse is true if $\mathfrak{C}$ is a coseparable $A$-coring. If ${ }_{A} X$ and ${ }_{B} \Lambda$ are flat, and $\omega_{X, \Lambda}=\rho_{X} \otimes_{B} \Lambda-X \otimes_{A} \rho_{\Lambda}$ is pure as an A-linear map and $\omega_{\Lambda, X}=\rho_{\Lambda} \otimes_{A} X-\Lambda \otimes_{B} \rho_{X}$ is pure as a B-linear map (e.g. if $\mathfrak{c} X$ and $\mathfrak{D} \Lambda$ are coflat [8, 21.5] or $A$ and $B$ are von Neumann regular rings), or if $\mathfrak{C}$ and $\mathfrak{D}$ are coseparable, then (4) implies (1). The converse is true if $\mathfrak{C} X$ and $\mathfrak{D} \Lambda$ are coflat, or if $A$ and $B$ are von Neumann regular rings, or if $\mathfrak{C}$ and $\mathfrak{D}$ are coseparable. Finally, if $\mathfrak{C}$ and $\mathfrak{D}$ are coseparable, or if $X$ and $\Lambda$ are coflat on both sides, or if $A, B$ are von Neumann regular rings, then (1), (4) and (5) are equivalent.

Proof. The equivalence between (11) and (2) follows from [18, Proposition 4.2]. That (21) implies (3) is a consequence of [18, Proposition 3.4]. If $\mathfrak{C}$ is coseparable and we assume (3]) then, by Corollary 2.5. $h_{\mathfrak{C}}(\Lambda,-) \simeq-\square_{\mathfrak{C}} h_{\mathfrak{C}}(\Lambda, \mathfrak{C}) \simeq-\square_{\mathfrak{C}} X$. That (11) implies (44) follows from Lemma 2.6 by evaluating the unit and the counit of the adjunction at $\mathfrak{C}$ and $\mathfrak{D}$, respectively. Conversely, if we put $F=-\square_{\mathfrak{C}} X$ and $G=-\square_{\mathfrak{D}} \Lambda$, we have $G F \simeq-\square_{\mathfrak{C}}\left(X \square_{\mathfrak{D}} \Lambda\right)$ and $F G \simeq-\square_{\mathfrak{D}}\left(\Lambda \square_{\mathfrak{C}} X\right)$ by [8, Proposition 22.6]. Define natural transformations

$$
\eta: 1_{\mathcal{M} \mathfrak{c}} \stackrel{\simeq}{\longrightarrow}-\square_{\mathfrak{C}} \mathfrak{C} \stackrel{-\square_{\mathfrak{C}} \psi}{\longrightarrow} G F
$$

and

$$
\varepsilon: F G \stackrel{-\square_{\mathfrak{D}} \omega}{\longrightarrow}-\square_{\mathfrak{D}} \mathfrak{D} \stackrel{\simeq}{\longrightarrow} 1_{\mathcal{M}^{\mathfrak{D}}}
$$

which become the unit and the counit of an adjunction by (41). This gives the equivalence between (11) and (41). The equivalence between (41) and (51) follows by symmetry.

Definition 2.8. Following [2] and [8], a bicomodule $N \in{ }^{\mathfrak{C}} \mathcal{M}^{\mathfrak{D}}$ is called an injector as a right $\mathfrak{D}$-comodule if the functor $-\otimes_{A} N: \mathcal{M}^{A} \rightarrow \mathcal{M}^{\mathfrak{D}}$ preserves injective objects.

Proposition 2.9. Suppose that ${ }_{A} \mathfrak{C}$ and ${ }_{B} \mathfrak{D}$ are flat. Let $X \in{ }^{\mathfrak{C}} \mathcal{M}^{\mathfrak{D}}$ and $\Lambda \in \mathfrak{D}^{\mathfrak{C}}$. The following statements are equivalent

(i) $-\square_{\mathfrak{C}} X$ is left adjoint to $-\square_{\mathfrak{D}} \Lambda$, and $-\square_{\mathfrak{C}} X$ is left exact (or ${ }_{A} X$ is flat or $\mathfrak{C} X$ is coflat);

(ii) $\Lambda$ is quasi-finite as a right $\mathfrak{C}$-comodule, $-\square_{\mathfrak{C}} X \simeq \mathrm{h}_{\mathfrak{C}}(\Lambda,-)$, and $-\square_{\mathfrak{C}} X$ is left exact (or ${ }_{A} X$ is flat or $\mathfrak{C} X$ is coflat); 
(iii) $\Lambda$ is quasi-finite and injector as a right $\mathfrak{C}$-comodule and $X \simeq \mathrm{h}_{\mathfrak{C}}(\Lambda, \mathfrak{C})$ in ${ }^{\mathfrak{c}} \mathcal{M}^{\mathcal{D}}$.

Proof. First, observe that if $\mathfrak{C}_{\mathfrak{C}} X$ is coflat, then ${ }_{A} X$ is flat [8, 21.6], and that if ${ }_{A} X$ is flat, then the functor $-\square_{\mathfrak{C}} X$ is left exact. Thus, in view of Proposition 2.7, it suffices if we prove that the version of $(i i)$ with $-\square_{\mathfrak{C}} X$ left exact implies (iii), and this last implies the version of (ii) with $\mathfrak{c} X$ coflat. Assume that $-\square_{\mathfrak{C}} X \simeq \mathrm{h}_{\mathfrak{C}}(\Lambda,-)$ with $-\square_{\mathfrak{C}} X$ left exact. By [18, Proposition 3.4], $X \simeq \mathrm{h}_{\mathfrak{C}}(\Lambda, \mathfrak{C})$ in ${ }^{\mathfrak{C}} \mathcal{M}^{\mathfrak{D}}$. Being a left adjoint, $\mathrm{h}_{\mathfrak{C}}(\Lambda,-)$ is right exact and, henceforth, exact. By [31, Theorem 3.2.8], $\Lambda_{\mathfrak{C}}$ is an injector and we have proved (iii). Conversely, if $\Lambda_{\mathfrak{C}}$ is a quasi-finite injector and $X \simeq \mathrm{h}_{\mathfrak{C}}(\Lambda, \mathfrak{C})$ as bicomodules, then $-\square_{\mathfrak{C}} X \simeq-\square_{\mathfrak{C}} h_{\mathfrak{C}}(\Lambda, \mathfrak{C})$ and, by [31, Theorem 3.2.8], we get that $h_{\mathfrak{C}}(\Lambda,-)$ is an exact functor. By Corollary 2.5, $h_{\mathfrak{C}}(\Lambda,-) \simeq-\square_{\mathfrak{C}} h_{\mathfrak{C}}(\Lambda, \mathfrak{C}) \simeq-\square_{\mathfrak{C}} X$, and $\mathfrak{C}_{\mathfrak{C}} X$ is coflat.

From the foregoing propositions, it is easy to deduce our characterization of Frobenius functors between categories of comodules over corings.

Theorem 2.10. Suppose that ${ }_{A} \mathfrak{C}$ and ${ }_{B} \mathfrak{D}$ are flat. Let $X \in{ }^{\mathfrak{C}} \mathcal{M}^{\mathfrak{D}}$ and $\Lambda \in{ }^{\mathfrak{D}} \mathcal{M}^{\mathfrak{C}}$. The following statements are equivalent

(i) $\left(-\square_{\mathfrak{C}} X,-\square_{\mathfrak{D}} \Lambda\right)$ is a Frobenius pair;

(ii) $-\square_{\mathfrak{C}} X$ is a Frobenius functor, and $\mathrm{h}_{\mathfrak{D}}(X, \mathfrak{D}) \simeq \Lambda$ as bicomodules;

(iii) there is a Frobenius pair $(F, G)$ for $\mathcal{M}^{\mathfrak{C}}$ and $\mathcal{M}^{\mathfrak{D}}$ such that $F(\mathfrak{C}) \simeq \Lambda$ and $G(\mathfrak{D}) \simeq X$ as bicomodules;

(iv) $\Lambda_{\mathfrak{C}}, X_{\mathfrak{D}}$ are quasi-finite injectors, and $X \simeq \mathrm{h}_{\mathfrak{C}}(\Lambda, \mathfrak{C})$ and $\Lambda \simeq \mathrm{h}_{\mathfrak{D}}(X, \mathfrak{D})$ as bicomodules;

(v) $\Lambda_{\mathfrak{C}}, X_{\mathfrak{D}}$ are quasi-finite, and $-\square_{\mathfrak{C}} X \simeq \mathrm{h}_{\mathfrak{C}}(\Lambda,-)$ and $-\square_{\mathfrak{D}} \Lambda \simeq \mathrm{h}_{\mathfrak{D}}(X,-)$.

Proof. $($ i $) \Leftrightarrow($ ii $) \Leftrightarrow($ iii) This is obvious, after Theorem [2.3 and [18, Proposition 3.4].

$(i) \Leftrightarrow(i v)$ Follows from Proposition 2.9.

(iv) $\Leftrightarrow(v)$ If $X_{\mathfrak{D}}$ and $\Lambda_{\mathfrak{C}}$ are quasi-finite, then ${ }_{A} X$ and ${ }_{B} \Lambda$ are flat. Now, apply Proposition 2.9]

From Proposition 2.7 and Proposition 2.9 (or Theorem 2.10) we get the following

Theorem 2.11. Let $X \in \mathfrak{C}^{\mathfrak{C}} \mathcal{M}^{\mathfrak{D}}$ and $\Lambda \in{ }^{\mathfrak{D}} \mathcal{M}^{\mathfrak{C}}$. Suppose that ${ }_{A} \mathfrak{C}, \mathfrak{C}_{A},{ }_{B} \mathfrak{D}$ and $\mathfrak{D}_{B}$ are flat. The following statements are equivalent

1. $\left(-\square_{\mathfrak{C}} X,-\square_{\mathfrak{D}} \Lambda\right)$ is a Frobenius pair, with $X_{\mathfrak{D}}$ and $\Lambda_{\mathfrak{C}}$ coflat;

2. $\left(\Lambda \square_{\mathfrak{C}}-, X \square_{\mathfrak{D}^{-}}\right)$is a Frobenius pair, with $\mathfrak{C} X$ and ${ }_{\mathfrak{D}} \Lambda$ coflat;

3. $X$ and $\Lambda$ are coflat quasi-finite injectors on both sides, and $X \simeq \mathrm{h}_{\mathfrak{C}}(\Lambda, \mathfrak{C})$ in ${ }^{\mathfrak{c}} \mathcal{M}^{\mathcal{D}}$ and $\Lambda \simeq \mathrm{h}_{\mathfrak{D}}(X, \mathfrak{D})$ in ${ }^{\mathfrak{D}} \mathcal{M}^{\mathfrak{C}}$. 
If moreover $\mathfrak{C}$ and $\mathfrak{D}$ are coseparable (resp. $A$ and $B$ are von Neumann regular rings), then the following statements are equivalent

1. $\left(-\square_{\mathfrak{C}} X,-\square_{\mathfrak{D}} \Lambda\right)$ is a Frobenius pair;

2. $\left(\Lambda \square_{\mathfrak{C}^{-}}, X \square_{\mathcal{D}^{-}}\right)$is a Frobenius pair;

3. $X$ and $\Lambda$ are quasi-finite (resp. quasi-finite injectors) on both sides, and $X \simeq \mathrm{h}_{\mathfrak{C}}(\Lambda, \mathfrak{C})$ in ${ }^{\mathfrak{C}} \mathcal{M}^{\mathfrak{D}}$ and $\Lambda \simeq \mathrm{h}_{\mathfrak{D}}(X, \mathfrak{D})$ in ${ }^{\mathfrak{D}} \mathcal{M}^{\mathfrak{C}}$.

Remark 2.12. In the case of rings (i.e., $\mathfrak{C}=A$ and $\mathfrak{D}=B$, Theorem 2.10 and the second part of Theorem 2.11 give [13, Theorem 2.1]. To see this, observe that ${ }_{A} X_{B}$ is quasi-finite as a right $B$-module if and only if $-\otimes_{A} X: \mathcal{M}_{A} \rightarrow \mathcal{M}_{B}$ has a left adjoint, that is, if and only if ${ }_{A} X$ is finitely generated and projective. In such a case, the left adjoint is $-\otimes_{B} \operatorname{Hom}_{A}(X, A): \mathcal{M}_{B} \rightarrow \mathcal{M}_{A}$. Of course, $-\square_{A} X=-\otimes_{A} X$.

The dual characterization in the framework of coalgebras over fields [13, Theorem 3.3] will be deduced in Section 3 (see Remarks 3.12).

\section{Frobenius functors between corings with a duality}

We will look to Frobenius functors for corings closer to coalgebras over fields, in the sense that the categories of comodules share a fundamental duality.

An object $M$ of a Grothendieck category $\mathbf{C}$ is said to be finitely generated [33, p. 121] if whenever $M=\sum_{i} M_{i}$ is a direct union of subobjects $M_{i}$, then $M=M_{i_{0}}$ for some index $i_{0}$. Alternatively, $M$ is finitely generated if the functor $\operatorname{Hom}_{\mathbf{C}}(M,-)$ preserves direct unions [33. Proposition V.3.2]. The category $\mathbf{C}$ is locally finitely generated if it has a family of finitely generated generators. Recall from [33, p. 122] that a finitely generated object $M$ is finitely presented if every epimorphism $L \rightarrow M$ with $L$ finitely generated has finitely generated kernel. By [33, Proposition V.3.4], if $\mathbf{C}$ is locally finitely generated, then $M$ is finitely presented if and only if $\operatorname{Hom}_{\mathbf{C}}(M,-)$ preserves direct limits. For the notion of a locally projective module we refer to [38].

Lemma 3.1. Let $\mathfrak{C}$ be a coring over a ring $A$ such that ${ }_{A} \mathfrak{C}$ is flat.

(1) A comodule $M \in \mathcal{M}^{\mathfrak{C}}$ is finitely generated if and only if $M_{A}$ is finitely generated.

(2) A comodule $M \in \mathcal{M}^{\mathfrak{C}}$ is finitely presented if $M_{A}$ is finitely presented. The converse is true whenever $\mathcal{M}^{\mathfrak{C}}$ is locally finitely generated.

(3) If ${ }_{A} \mathfrak{C}$ is locally projective, then $\mathcal{M}^{\mathfrak{C}}$ is locally finitely generated.

Proof. The forgetful functor $U: \mathcal{M}^{\mathfrak{c}} \rightarrow \mathcal{M}_{A}$ has an exact left adjoint $-\otimes_{A} \mathfrak{C}: \mathcal{M}_{A} \rightarrow \mathcal{M}^{\mathfrak{C}}$ which preserves direct limits. Thus, $U$ preserves finitely generated objects and, in case that $\mathcal{M}^{\mathfrak{C}}$ is locally finitely generated, finitely presented objects. Now, if $M \in \mathcal{M}^{\mathfrak{C}}$ is finitely generated as a right $A$-module, and $M=\sum_{i} M_{i}$ as a direct union of subcomodules, then 
$U(M)=U\left(\sum_{i} M_{i}\right)=\sum_{i} U\left(M_{i}\right)$, since $U$ is exact and preserves coproducts. Therefore, $U(M)=U\left(M_{i_{0}}\right)$ for some index $i_{0}$ which implies, being $U$ a faithfully exact functor, that $M=M_{i_{0}}$. Thus, $M$ is a finitely generated comodule. We have thus proved (11), and the converse to (2). Now, if $M \in \mathcal{M}^{\mathfrak{C}}$ is such that $M_{A}$ is finitely presented, then for every exact sequence $0 \rightarrow K \rightarrow L \rightarrow M \rightarrow 0$ in $\mathcal{M}^{\mathfrak{C}}$ with $L$ finitely generated, we get an exact sequence $0 \rightarrow K_{A} \rightarrow L_{A} \rightarrow M_{A} \rightarrow 0$ with $M_{A}$ finitely presented. Thus, $K_{A}$ is finitely generated and, by (II), $K \in \mathcal{M}^{\mathfrak{C}}$ is finitely generated. This proves that $M$ is a finitely presented comodule. Finally, (3) is a consequence of (11) and [ㅇ, 19.12(1)].

The notation $\mathbf{C}_{f}$ stands for the full subcategory of a Grothendieck category $\mathbf{C}$ whose objects are the finitely generated objects. The category $\mathbf{C}$ is locally noetherian 33 . $\mathrm{p}$. 123] if it has a family of noetherian generators or equivalently, if $\mathbf{C}$ is locally finitely generated and every finitely generated object of $\mathbf{C}$ is noetherian. By 33, Proposition V.4.2, Proposition V.4.1, Lemma V.3.1(i)], in an arbitrary Grothendieck category, every finitely generated object is noetherian if and only if every finitely generated object is finitely presented. The version for categories of modules of the following result is well-known.

Lemma 3.2. Let $\mathbf{C}$ be a locally finitely generated category.

(1) The category $\mathbf{C}_{f}$ is additive.

(2) The category $\mathbf{C}_{f}$ has cokernels, and every monomorphism in $\mathbf{C}_{f}$ is a monomorphism in $\mathbf{C}$.

(3) The following statements are equivalent:

(a) The category $\mathbf{C}_{f}$ has kernels;

(b) $\mathbf{C}$ is locally noetherian;

(c) $\mathbf{C}_{f}$ is abelian;

(d) $\mathbf{C}_{f}$ is an abelian subcategory of $\mathbf{C}$.

Proof. (1) Straightforward.

(2) That $\mathbf{C}_{f}$ has cokernels is straightforward from [33, Lemma V.3.1(i)]. Now, let $f: M \rightarrow N$ be a monomorphism in $\mathbf{C}_{f}$ and $\xi: X \rightarrow M$ be a morphism in $\mathbf{C}$ such that $f \xi=0$. Suppose that $X=\bigcup_{i \in I} X_{i}$, where $X_{i} \in \mathbf{C}_{f}$, and $\iota_{i}: X_{i} \rightarrow X, i \in I$ the canonical injections. Then $f \xi \iota_{i}=0$, and $\xi \iota_{i}=0$, for every $i$, and by the definition of the inductive limit, $\xi=0$.

(3) $(b) \Rightarrow(a)$ Straightforward from [33, Proposition V.4.1].

$(d) \Rightarrow(c)$ and $(c) \Rightarrow(a)$ are trivial.

$(a) \Rightarrow(b)$ Let $M \in \mathbf{C}_{f}$, and $K$ be a subobject of $M$. Let $\iota: L \rightarrow M$ the kernel of the canonical morphism $f: M \rightarrow M / K$ in $\mathbf{C}_{f}$. Suppose that $K=\bigcup_{i \in I} K_{i}$, where $K_{i} \in \mathbf{C}_{f}$, for every $i \in I$. By the universal property of the kernel, there exist a unique morphism 
$\alpha: L \rightarrow K$, and a unique morphism $\beta_{i}: K_{i} \rightarrow L$, for every $i \in I$, making commutative the diagrams

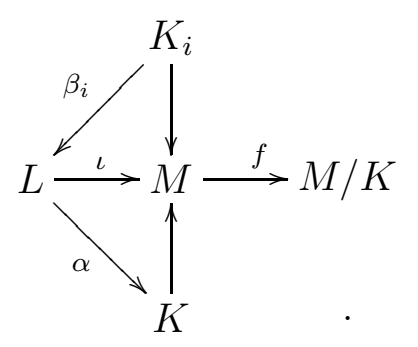

By (2), $\iota$ is a monomorphism in $\mathbf{C}$, then for every $K_{i} \subset K_{j}$, the diagram

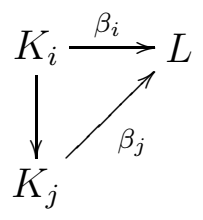

commutes. Therefore we have the commutative diagram

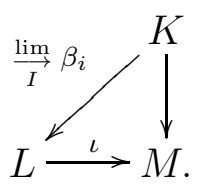

Then $K \simeq L$, and hence $K \in \mathbf{C}_{f}$. Finally, by [33, Proposition V.4.1], $M$ is noetherian in C.

$(b) \Rightarrow(d)$ Straightforward from [17, Theorem 3.41].

The following generalization of [13, Proposition 3.1] will allow us to give an alternative proof to the equivalence " $(1) \Leftrightarrow(4)$ " of Theorem 3.11 (see Remarks 3.12).

Proposition 3.3. Let $\mathbf{C}$ and $\mathbf{D}$ be two locally noetherian categories. Then

(1) If $F: \mathbf{C} \rightarrow \mathbf{D}$ is a Frobenius functor, then its restriction $F_{f}: \mathbf{C}_{f} \rightarrow \mathbf{D}_{f}$ is a Frobenius functor.

(2) If $H: \mathbf{C}_{f} \rightarrow \mathbf{D}_{f}$ is a Frobenius functor, then $H$ can be uniquely extended to a Frobenius functor $\bar{H}: \mathbf{C} \rightarrow \mathbf{D}$.

(3) The assignment $F \mapsto F_{f}$ defines a bijective correspondence (up to natural isomorphisms) between Frobenius functors from $\mathbf{C}$ to $\mathbf{D}$ and Frobenius functors from $\mathbf{C}_{f}$ to $\mathbf{D}_{f}$.

(4) In particular, if $\mathbf{C}=\mathcal{M}^{\mathfrak{C}}$ and $\mathbf{D}=\mathcal{M}^{\mathfrak{D}}$ are locally noetherian such that ${ }_{A} \mathfrak{C}$ and ${ }_{B} \mathfrak{D}$ are flat, then $F: \mathcal{M}^{\mathfrak{C}} \rightarrow \mathcal{M}^{\mathfrak{D}}$ is a Frobenius functor if and only if it preserves direct limits and comodules which are finitely generated as right A-modules, and the restriction functor $F_{f}: \mathcal{M}_{f}^{\mathfrak{C}} \rightarrow \mathcal{M}_{f}^{\mathfrak{D}}$ is a Frobenius functor. 
Proof. The proofs of [13, Proposition 3.1 and Remark 3.2] remain valid for our situation, but with some minor modifications: to prove that $\bar{H}$ is well-defined, we use Lemma 3.2 . In the proof of the statements (1), (2) and (3) we use the Grothendieck AB 5 condition.

In order to generalize [35, Proposition A.2.1] and its proof, we need the following lemma.

Lemma 3.4. (1) Let $\mathbf{C}$ be a locally noetherian category, let $\mathbf{D}$ be an arbitrary Grothendieck category, $F: \mathbf{C} \rightarrow \mathbf{D}$ be an arbitrary functor which preserves direct limits, and $F_{f}: \mathbf{C}_{f} \rightarrow \mathbf{D}$ be its restriction to $\mathbf{C}_{f}$. Then $F$ is exact (faithfully exact, resp. left, right exact) if and only if $F_{f}$ is exact (faithfully exact, resp. left, right exact).

In particular, an object $M$ in $\mathbf{C}_{f}$ is projective (resp. projective generator) if and only if it is projective (resp. projective generator) in $\mathbf{C}$.

(2) Let $\mathbf{C}$ be a locally noetherian category. For every object $M$ of $\mathbf{C}$, the following conditions are equivalent

(a) $M$ is injective (resp. an injective cogenerator);

(b) the contravariant functor $\operatorname{Hom}_{\mathbf{C}}(-, M): \mathbf{C} \rightarrow \mathbf{A b}$ is exact (resp. faithfully exact);

(c) the contravariant functor $\operatorname{Hom}_{\mathbf{C}}(-, M)_{f}: \mathbf{C}_{f} \rightarrow \mathbf{A b}$ is exact (resp. faithfully exact).

In particular, an object $M$ in $\mathbf{C}_{f}$ is injective (resp. injective cogenerator) if and only if it is injective (resp. injective cogenerator) in $\mathbf{C}$.

Proof. (1) The "only if" part is straightforward from the fact that the injection functor $\mathbf{C}_{f} \rightarrow \mathbf{C}$ is faithfully exact.

For the "if" part, suppose that $F_{f}$ is left exact. Let $f: M \rightarrow N$ be a morphism in C. Put $M=\bigcup_{i \in I} M_{i}$ and $N=\bigcup_{j \in J} N_{j}$, as direct union of directed families of finitely generated subobjects. For $(i, j) \in I \times J$, let $M_{i, j}=M_{i} \cap f^{-1}\left(N_{j}\right)$, and $f_{i, j}: M_{i, j} \rightarrow N_{j}$ be the restriction of $f$ to $M_{i, j}$. We have $f=\underset{\overrightarrow{I \times J}}{\lim f_{i, j}}$ and then $F(f)=\underset{\overrightarrow{I \times J}}{\lim _{\vec{X}} F_{f}}\left(f_{i, j}\right)$. Hence

$\operatorname{ker} F(f)=\operatorname{ker} \underset{I \times J}{\lim } F_{f}\left(f_{i, j}\right)=\underset{I \times J}{\lim } \operatorname{ker} F_{f}\left(f_{i, j}\right)=\underset{\underset{I \times J}{\longrightarrow}}{\lim F_{f}}\left(\operatorname{ker} f_{i, j}\right)=\underset{I \times J}{\lim F}\left(\operatorname{ker} f_{i, j}\right)$ (by Lemma 3.2$)=F\left(\underset{I \times J}{\lim } \operatorname{ker} f_{i, j}\right)=F(\operatorname{ker} f)$.

Finally $F$ is left exact. Analogously, it can be proved that $F_{f}$ is right exact implies that $F$ is also right exact. Now, suppose that $F_{f}$ is faithfully exact. We have already proved that $F$ is exact. It remains to prove that $F$ is faithful. For this, let $0 \neq M=\bigcup_{i \in I} M_{i}$ be an object of $\mathbf{C}$, where $M_{i}$ is finitely generated for every $i \in I$. We have

$$
F(M)=\underset{I}{\lim } F_{f}\left(M_{i}\right) \simeq \sum_{i} F_{f}\left(M_{i}\right)
$$

(since $F$ is exact). Since $M \neq 0$, there exists some $i_{0} \in I$ such that $M_{i_{0}} \neq 0$. By 33 , Proposition IV.6.1], $F_{f}\left(M_{i_{0}}\right) \neq 0$, hence $F(M) \neq 0$. Also by [33, Proposition IV.6.1], $F$ is faithful. 
(2) $(a) \Leftrightarrow(b)$ Obvious.

$(b) \Rightarrow(c)$ Analogous to that of the "only if" part of (1).

$(c) \Rightarrow(a)$ That $M$ is injective is a consequence of 33, Proposition V.2.9, Proposition V.4.1]. Now, suppose moreover that $\operatorname{Hom}_{\mathbf{C}}(-, M)_{f}$ is faithful. Let $L$ be a non-zero object of $\mathbf{C}$, and $K$ be a non-zero finitely generated subobject of $L$. By [33, Proposition IV.6.1], there exists a non-zero morphism $K \rightarrow M$. Since $M$ is injective, there exists a non-zero morphism $L \rightarrow M$ making commutative the following diagram

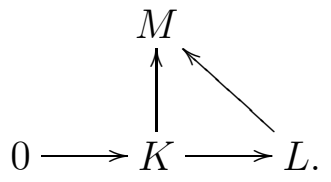

From [33, Proposition IV.6.5], it follows that $M$ is a cogenerator.

If $\mathfrak{C}_{A}$ is flat and $M \in \mathcal{M}^{\mathfrak{C}}$ is finitely presented as right $A$-module, then [8, 19.19] the dual left $A$-module $M^{*}=\operatorname{Hom}_{A}(M, A)$ has a left $\mathfrak{C}$-comodule structure

$$
M^{*} \simeq \operatorname{Hom}_{\mathfrak{C}}(M, \mathfrak{C}) \subseteq \operatorname{Hom}_{A}(M, \mathfrak{C}) \simeq \mathfrak{C} \otimes_{A} M^{*}
$$

Now, if ${ }_{A} M^{*}$ turns out to be finitely presented and ${ }_{A} \mathfrak{C}$ is flat, then ${ }^{*}\left(M^{*}\right)=\operatorname{Hom}_{A}\left(M^{*}, A\right)$ is a right $\mathfrak{C}$-comodule and the canonical map $\sigma_{M}: M \rightarrow^{*}\left(M^{*}\right)$ is a homomorphism in $\mathcal{M}^{\mathfrak{C}}$. This construction leads to a duality (i.e. a contravariant equivalence)

$$
(-)^{*}: \mathcal{M}_{0}^{\mathfrak{C}} \leftrightarrows{ }^{\mathfrak{C}} \mathcal{M}_{0}:{ }^{*}(-)
$$

between the full subcategories $\mathcal{M}_{0}^{\mathfrak{C}}$ and ${ }^{\mathfrak{c}} \mathcal{M}_{0}$ of $\mathcal{M}^{\mathfrak{c}}$ and ${ }^{\mathfrak{C}} \mathcal{M}$ whose objects are the comodules which are finitely generated and projective over $A$ on the corresponding side (this holds even without flatness assumptions of $\mathfrak{C}$ ). Call it the basic duality (details may be found in [10]). Of course, in the case that $A$ is semisimple (e.g. for coalgebras over fields) these categories are that of finitely generated comodules, and this basic duality plays a remarkable role in the study of several notions in the coalgebra setting (e.g. Morita equivalence [36, semiperfect coalgebras [23, Morita duality [19, 20, or Frobenius Functors [13]). It would be interesting to know, in the coring setting, to what extent the basic duality can be extended to the subcategories $\mathcal{M}_{f}^{\mathfrak{c}}$ and ${ }^{\mathfrak{C}} \mathcal{M}_{f}$, since, as we will try to show in this section, this allows to obtain better results. Of course, this is the underlying idea when the ground $\operatorname{ring} A$ is assumed to be Quasi-Frobenius (see [15] for the case of semiperfect corings and Morita duality), but we hope future developments of the theory will be aided by the more general setting we propose here.

Consider contravariant functors between Grothendieck categories $H: \mathbf{A} \leftrightarrows \mathbf{A}^{\prime}: H^{\prime}$, together with natural transformations $\tau: 1_{\mathbf{A}} \rightarrow H^{\prime} \circ H$ and $\tau^{\prime}: 1_{\mathbf{A}^{\prime}} \rightarrow H \circ H^{\prime}$, satisfying the condition $H\left(\tau_{X}\right) \circ \tau_{H(X)}^{\prime}=1_{H(X)}$ and $H^{\prime}\left(\tau_{X^{\prime}}^{\prime}\right) \circ \tau_{H^{\prime}\left(X^{\prime}\right)}=1_{H^{\prime}\left(X^{\prime}\right)}$ for $X \in \mathbf{A}$ and $X^{\prime} \in \mathbf{A}^{\prime}$. Following [14], this situation is called a right adjoint pair.

Proposition 3.5. Let $\mathfrak{C}$ be an $A$-coring such that ${ }_{A} \mathfrak{C}$ and $\mathfrak{C}_{A}$ are flat. Assume that $\mathcal{M}^{\mathfrak{C}}$ and $\mathfrak{C}^{\mathfrak{M}}$ are locally noetherian categories. If ${ }_{A} M^{*}$ and ${ }^{*} N_{A}$ are finitely generated modules 
for every $M \in \mathcal{M}_{f}^{\mathfrak{C}}$ and $N \in \mathfrak{C}^{\mathfrak{C}} \mathcal{M}_{f}$, then the basic duality extends to a right adjoint pair $(-)^{*}: \mathcal{M}_{f}^{\mathfrak{c}} \leftrightarrows{ }^{\mathfrak{C}} \mathcal{M}_{f}:{ }^{*}(-)$

Proof. If $M \in \mathcal{M}_{f}^{\mathfrak{c}}$ then, since $\mathcal{M}^{\mathfrak{c}}$ is locally noetherian, $M_{\mathfrak{C}}$ is finitely presented. By Lemma 3.1. $M_{A}$ is finitely presented and the left $\mathfrak{C}$-comodule $M^{*}$ makes sense. Now, the assumption ${ }_{A} M^{*}$ finitely generated implies, by Lemma 3.1. that $M^{*} \in{ }^{\mathfrak{C}} \mathcal{M}_{f}$. We have then the functor $(-)^{*}: \mathcal{M}_{f}^{\mathfrak{c}} \rightarrow{ }^{\mathfrak{C}} \mathcal{M}_{f}$. The functor $(-)^{*}$ is analogously defined, and the rest of the proof consists of straightforward verifications.

Example 3.6. The hypotheses are fulfilled if ${ }_{A} \mathfrak{C}$ and $\mathfrak{C}_{A}$ are locally projective and $A$ is left and right noetherian (in this case the right adjoint pair already appears in [15]). But there are situations in which no finiteness condition need to be required to $A$ : this is the case, for instance, of cosemisimple corings (see [16, Theorem 3.1]). In particular, if an arbitrary ring $A$ contains a division ring $B$, then, by [16. Theorem 3.1] the canonical coring $A \otimes_{B} A$ satisfies all hypotheses in Proposition 3.5.

Definition 3.7. Let $\mathfrak{C}$ be a coring over $A$ satisfying the assumptions of Proposition 3.5. We will say that $\mathfrak{C}$ has a duality if the basic duality extends to a duality

$$
(-)^{*}: \mathcal{M}_{f}^{\mathfrak{c}} \leftrightarrows{ }^{\mathfrak{C}} \mathcal{M}_{f}:{ }^{*}(-)
$$

We have the following examples of a coring which has a duality:

- $\mathfrak{C}$ is a coring over a $\mathrm{QF}$ ring $A$ such that ${ }_{A} \mathfrak{C}$ and $\mathfrak{C}_{A}$ are flat (and hence projective);

- $\mathfrak{C}$ is a cosemisimple coring; where, by [16, Theorem 3.1], $\mathcal{M}_{f}^{\mathfrak{c}}$ and ${ }^{\mathfrak{C}} \mathcal{M}_{f}$ are equal to $\mathcal{M}_{0}^{\mathfrak{E}}$ and $\mathfrak{C}^{\mathfrak{c}} \mathcal{M}_{0}$, respectively;

- $\mathfrak{C}$ is a coring over $A$ such that ${ }_{A} \mathfrak{C}$ and $\mathfrak{C}_{A}$ are flat and semisimple, $\mathcal{M}^{\mathfrak{c}}$ and ${ }^{\mathfrak{c}} \mathcal{M}$ are locally noetherian categories, and the dual of every simple right (resp. left) $A$-module in the decomposition of $\mathfrak{C}_{A}$ (resp. ${ }_{A} \mathfrak{C}$ ) as a direct sum of simple $A$-modules is finitely generated and $A$-reflexive (in fact, every right (resp. left) $\mathfrak{C}$-comodule $M$ becomes a submodule of the semisimple right (resp. left) $A$-module $M \otimes_{A} \mathfrak{C}$, and hence $M_{A}$ (resp. $\left.{ }_{A} M\right)$ is also semisimple).

Proposition 3.8. Suppose that the coring $\mathfrak{C}$ has a duality. Let $M \in \mathcal{M}^{\mathfrak{C}}$ such that $M_{A}$ is flat. The following are equivalent

1. $M$ is coflat (resp. faithfully coflat);

2. $\operatorname{Hom}_{\mathfrak{C}}(-, M)_{f}: \mathcal{M}_{f}^{\mathfrak{c}} \rightarrow \mathcal{M}_{k}$ is exact (resp. faithfully exact);

3. $M$ is injective (resp. an injective cogenerator). 
Proof. Let $M \in \mathcal{M}^{\mathfrak{C}}$ and $N \in{ }^{\mathfrak{C}} \mathcal{M}_{f}$. We have the following commutative diagram (in $\mathcal{M}_{k}$ )

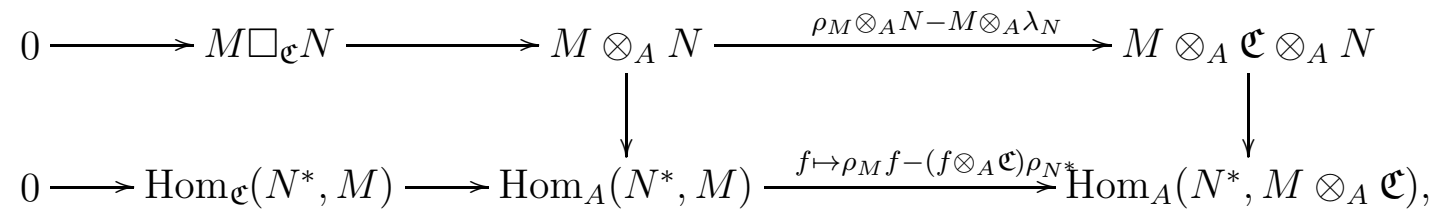

where the vertical maps are the canonical maps. By the universal property of the kernel, there is a unique morphism $\eta_{M, N}: M \square_{\mathfrak{C}} N \rightarrow \operatorname{Hom}_{\mathfrak{C}}\left(N^{*}, M\right)$ making commutative the above diagram. By the cube Lemma (see [26, Proposition II.1.1]), $\eta$ is a natural transformation of bifunctors. If $M_{A}$ is flat then $\eta_{M, N}$ is an isomorphism for every $N \in{ }^{\mathfrak{c}} \mathcal{M}_{f}$. We have

$$
M \square_{\mathfrak{C}}-\simeq \operatorname{Hom}_{\mathfrak{C}}(-, M)_{f} \circ(-)^{*}:{ }^{\mathfrak{C}} \mathcal{M}_{f} \rightarrow \mathcal{M}_{k}
$$

Then, by Lemma 3.4, $M_{\mathfrak{C}}$ is coflat (resp. faithfully coflat) iff $M \square_{\mathfrak{C}}-:{ }^{\mathfrak{c}} \mathcal{M}_{f} \rightarrow \mathcal{M}_{k}$ is exact (resp. faithfully exact) iff $\operatorname{Hom}_{\mathfrak{C}}(-, M)_{f}: \mathcal{M}_{f}^{\mathfrak{C}} \rightarrow \mathcal{M}_{k}$ is exact (resp. faithfully exact) iff $M_{\mathfrak{C}}$ is injective (resp. an injective cogenerator).

The particular case of the following result for coalgebras over commutative ring is given in $[2$.

Corollary 3.9. Let $N \in \mathfrak{C}^{\mathfrak{C}} \mathcal{M}^{\mathfrak{D}}$ be a bicomodule. Suppose that $A$ is a $Q F$ ring and $\mathfrak{D}$ has a duality. If $N$ is injective in $\mathcal{M}^{\mathfrak{D}}$ such that $N_{B}$ is flat, then $N$ is an injector as a right $\mathfrak{D}$-comodule.

Proof. Let $X_{A}$ be an injective module. Since $A$ is a QF ring, $X_{A}$ is projective. We have then the natural isomorphism

$$
\left(X \otimes_{A} N\right) \square_{\mathfrak{D}}-\simeq X \otimes_{A}\left(N \square_{\mathfrak{D}}-\right):{ }^{\mathfrak{D}} \mathcal{M} \rightarrow \mathcal{M}_{k}
$$

By Proposition 3.8, $N_{\mathfrak{D}}$ and $X_{A}$ are coflat, and then $X \otimes_{A} N$ is coflat. Now, since $X \otimes_{A} N$ is a flat right $B$-module, and by Proposition [3.8, $X \otimes_{A} N$ is injective in $\mathcal{M}^{\mathfrak{D}}$.

The last two results allow to improve our general statements in Section 2 for corings having a duality.

Proposition 3.10. Suppose that $\mathfrak{C}$ and $\mathfrak{D}$ have a duality. Consider the following statements

1. $\left(-\square_{\mathfrak{C}} X,-\square_{\mathfrak{D}} \Lambda\right)$ is an adjoint pair of functors, with ${ }_{A} X$ and $\Lambda_{A}$ flat;

2. $\Lambda$ is quasi-finite injective as a right $\mathfrak{C}$-comodule, with ${ }_{A} X$ and $\Lambda_{A}$ flat and $X \simeq$ $\mathrm{h}_{\mathfrak{C}}(\Lambda, \mathfrak{C})$ in $\mathfrak{C}^{\mathfrak{D}}$.

We have (1) implies (2), and the converse is true if in particular B is a QF ring. 
Proof. (1) $\Rightarrow(2)$ From Proposition 3.8, $\mathfrak{D}$ is injective in $\mathcal{M}^{\mathfrak{D}}$. Since the functor $-\square_{\mathfrak{C}} X$ is exact, $\Lambda \simeq \mathfrak{D} \square_{\mathfrak{D}} \Lambda$ is injective in $\mathcal{M}^{\mathfrak{C}}$.

$(2) \Rightarrow(1)$ Assume that $B$ is a QF ring. From Corollary 3.9, $\Lambda$ is quasi-finite injector as a right $\mathfrak{C}$-comodule, and Proposition 2.9 achieves the proof.

We are now in a position to state and prove our main result of this section.

Theorem 3.11. Suppose that $\mathfrak{C}$ and $\mathfrak{D}$ have a duality. Let $X \in \mathfrak{C}^{\mathfrak{C}} \mathcal{M}^{\mathfrak{D}}$ and $\Lambda \in \mathfrak{D}^{\mathfrak{C}}$. The following statements are equivalent

1. $\left(-\square_{\mathfrak{C}} X,-\square_{\mathfrak{D}} \Lambda\right)$ is a Frobenius pair, with $X_{B}$ and $\Lambda_{A}$ flat;

2. $\left(\Lambda \square_{\mathfrak{C}^{-}}, X \square_{\mathfrak{D}^{-}}\right)$is a Frobenius pair, with ${ }_{A} X$ and ${ }_{B} \Lambda$ flat;

3. $X$ and $\Lambda$ are quasi-finite injector on both sides, and $X \simeq \mathrm{h}_{\mathfrak{C}}(\Lambda, \mathfrak{C})$ in ${ }^{\mathfrak{C}} \mathcal{M}^{\mathfrak{D}}$ and $\Lambda \simeq \mathrm{h}_{\mathfrak{D}}(X, \mathfrak{D})$ in ${ }^{\mathfrak{D}} \mathcal{M}^{\mathfrak{C}}$.

In particular, if $A$ and $B$ are $Q F$ rings, then the above statements are equivalent to

4. $X$ and $\Lambda$ are quasi-finite injective on both sides, and $X \simeq \mathrm{h}_{\mathfrak{C}}(\Lambda, \mathfrak{C})$ in ${ }^{\mathfrak{C}} \mathcal{M}^{\mathfrak{D}}$ and $\Lambda \simeq \mathrm{h}_{\mathfrak{D}}(X, \mathfrak{D})$ in ${ }^{\mathfrak{D}} \mathcal{M}^{\mathfrak{C}}$.

Finally, suppose that $\mathfrak{C}$ and $\mathfrak{D}$ are cosemisimple corings. Let $X \in{ }^{\mathfrak{C}} \mathcal{M}^{\mathfrak{D}}$ and $\Lambda \in \mathfrak{D}^{\mathfrak{D}} \mathcal{M}^{\mathfrak{C}}$. The following statements are equivalent

1. $\left(-\square_{\mathfrak{C}} X,-\square_{\mathfrak{D}} \Lambda\right)$ is a Frobenius pair;

2. $\left(\Lambda \square_{\mathfrak{C}-}, X \square_{\mathfrak{D}}-\right)$ is a Frobenius pair;

3. $X$ and $\Lambda$ are quasi-finite on both sides, and $X \simeq \mathrm{h}_{\mathfrak{C}}(\Lambda, \mathfrak{C})$ in ${ }^{\mathfrak{C}} \mathcal{M}^{\mathfrak{D}}$ and $\Lambda \simeq \mathrm{h}_{\mathfrak{D}}(X, \mathfrak{D})$ in ${ }^{\mathfrak{D}} \mathcal{M}^{\mathfrak{C}}$.

Proof. We start by proving the first part. In view of Theorem 2.11 and Theorem 2.10 it suffices to show that if $\left(-\square_{\mathfrak{C}} X,-\square_{\mathfrak{D}} \Lambda\right)$ is a Frobenius pair, the condition " $X_{\mathfrak{D}}$ and $\Lambda_{\mathfrak{C}}$ are coflat" is equivalent to " $X_{B}$ and $\Lambda_{A}$ are flat". Indeed, the first implication is obvious, for the converse, assume that $X_{B}$ and $\Lambda_{A}$ are flat. By Proposition 3.10, $X$ and $\Lambda$ are injective in $\mathcal{M}^{\mathfrak{D}}$ and $\mathcal{M}^{\mathfrak{C}}$ respectively, and they are coflat by Proposition 3.8. The particular case is straightforward from Proposition 3.10 and the above equivalences .

Now we will show the second part. We know that cosemisimple corings have a duality. By [16, Theorem 3.1], every comodule category over a cosemisimple coring is a spectral category (see [33, p. 128]). Thus, the bicomodules $\mathfrak{C}_{\mathfrak{D}} X_{\mathfrak{D}}$ and ${ }_{\mathfrak{D}} \Lambda_{\mathfrak{C}}$ are coflat and injector on both sides (we can see this directly by using the fact that every additive functor between abelian categories preserves split exactness). Now, apply the first part.

Remarks 3.12. $\quad$ 1. The equivalence " $(1) \Leftrightarrow(4)$ " of the last theorem is a generalization of [13, Theorem 3.3]. The proof of [13, Theorem 3.3] gives an alternative proof of "(1) $\Leftrightarrow(4)$ " of Theorem 3.11, using Proposition 3.3. 
2. The adjunction of Proposition 2.7 and Proposition 3.10] generalizes the coalgebra version of Morita's theorem [9, Theorem 4.2].

Example 3.13. Let $A$ be a $k$-algebra. Put $\mathfrak{C}=A$ and $\mathfrak{D}=k$. The bicomodule $A \in{ }^{\mathfrak{C}} \mathcal{M}^{\mathfrak{D}}$ is quasi-finite as a right $\mathfrak{D}$-comodule. $A$ is an injector as a right $\mathfrak{D}$-comodule if and only if the $k$-module $A$ is flat. If we take $A=k=\mathbb{Z}$, the bicomodule $A$ is quasi-finite and injector as a right $\mathfrak{D}$-comodule but it is not injective in $\mathcal{M}^{\mathfrak{D}}$. Hence, the assertion " $\left(-\square_{\mathfrak{C}} X,-\square_{\mathfrak{D}} \Lambda\right)$ is an adjoint pair of functors" does not imply in general the assertion " $\Lambda$ is quasi-finite injective as a right $\mathfrak{C}^{\mathfrak{c}}$-comodule and $X \simeq \mathrm{h}_{\mathfrak{C}}(\Lambda, \mathfrak{C})$ in ${ }^{\mathfrak{C}} \mathcal{M}^{\mathfrak{D}}$ ", and the following statements are not equivalent in general:

1. $\left(-\square_{\mathfrak{C}} X,-\square_{\mathfrak{D}} \Lambda\right)$ is a Frobenius pair;

2. $X$ and $\Lambda$ are quasi-finite injective on both sides, and $X \simeq \mathrm{h}_{\mathfrak{C}}(\Lambda, \mathfrak{C})$ in ${ }^{\mathfrak{C}} \mathcal{M}^{\mathfrak{D}}$ and $\Lambda \simeq \mathrm{h}_{\mathfrak{D}}(X, \mathfrak{D})$ in ${ }^{\mathfrak{D}} \mathcal{M}^{\mathfrak{C}}$.

On the other hand, there exists a commutative self-injective ring wich is not coherent. By a theorem of S.U. Chase (see for example [3, Theorem 19.20]), there exists then a $k$ algebra $A$ which is injective, but not flat as $k$-module. Hence, the bicomodule $A \in \mathfrak{C}^{\mathfrak{c}} \mathcal{M}^{\mathfrak{D}}$ is quasi-finite and injective as a right $\mathfrak{D}$-comodule, but not an injector as a right $\mathfrak{D}$-comodule.

\section{Applications to induction functors}

We start this section by recalling from [18, that a coring homomorphism from the coring $\mathfrak{C}$ into the coring $\mathfrak{D}$ is a pair $(\varphi, \rho)$, where $\rho: A \rightarrow B$ is a homomorphism of $k$-algebras and $\varphi: \mathfrak{C} \rightarrow \mathfrak{D}$ is a homomorphism of $A$-bimodules such that

$$
\epsilon_{\mathfrak{D}} \circ \varphi=\rho \circ \epsilon_{\mathfrak{C}} \quad \text { and } \quad \Delta_{\mathfrak{D}} \circ \varphi=\omega_{\mathfrak{D}, \mathfrak{D}} \circ\left(\varphi \otimes_{A} \varphi\right) \circ \Delta_{\mathfrak{C}},
$$

where $\omega_{\mathfrak{D}, \mathfrak{D}}: \mathfrak{D} \otimes_{A} \mathfrak{D} \rightarrow \mathfrak{D} \otimes_{B} \mathfrak{D}$ is the canonical map induced by $\rho: A \rightarrow B$.

Now we will characterize when the induction functor $-\otimes_{A} B: \mathcal{M}^{\mathfrak{C}} \rightarrow \mathcal{M}^{\mathfrak{D}}$ defined in [18, Proposition 5.3] is a Frobenius functor. The coaction of $\mathfrak{D}$ over $M \otimes_{A} B$ is given, when expressed in Sweedler's sigma notation, by

$$
\rho_{M \otimes_{A} B}\left(m \otimes_{A} b\right)=\sum m_{(0)} \otimes_{A} 1_{B} \otimes_{B} \varphi\left(m_{(1)}\right) b
$$

where $M$ is a right $\mathfrak{C}$-comodule with coaction $\rho_{M}(m)=\sum m_{(0)} \otimes_{A} m_{(1)}$. We also define the functor $-\square_{\mathfrak{D}}\left(B \otimes_{A} \mathfrak{C}\right): \mathcal{M}^{\mathfrak{D}} \rightarrow \mathcal{M}^{\mathfrak{C}}$, where the left coaction on the left $B$-module $B \otimes_{A} \mathfrak{C}$ is given by:

$$
\lambda_{B \otimes_{A} \mathfrak{C}}: B \otimes_{A} \mathfrak{C} \rightarrow \mathfrak{D} \otimes_{B} B \otimes_{A} \mathfrak{C} \simeq \mathfrak{D} \otimes_{A} \mathfrak{C}, \quad b \otimes_{A} c \mapsto \sum b \varphi\left(c_{(1)}\right) \otimes_{A} c_{(2)}
$$

where $\Delta_{\mathfrak{C}}(c)=\sum c_{(1)} \otimes_{A} c_{(2)}$. Moreover, if $\omega_{Y, B \otimes_{A} \mathfrak{C}}$ is ${ }_{A} \mathfrak{C}$-pure for every right $\mathfrak{D}$-comodule $Y$, then, by [18, Proposition 5.4], we have the adjoint pair of functors $\left(-\otimes_{A} B,-\square_{\mathfrak{D}}\left(B \otimes_{A}\right.\right.$ $\mathfrak{C}))$. 
Theorem 4.1. Let $(\varphi, \rho): \mathfrak{C} \rightarrow \mathfrak{D}$ be a homomorphism of corings such that ${ }_{A} \mathfrak{C}$ and ${ }_{B} \mathfrak{D}$ are flat. The following statements are equivalent

(a) $-\otimes_{A} B: \mathcal{M}^{\mathfrak{C}} \rightarrow \mathcal{M}^{\mathfrak{D}}$ is a Frobenius functor;

(b) the $\mathfrak{C}-\mathfrak{D}$-bicomodule $\mathfrak{C} \otimes_{A} B$ is quasi-finite and injector as a right $\mathfrak{D}$-comodule and there exists an isomorphism of $\mathfrak{D}-\mathfrak{C}$-bicomodules $\mathrm{h}_{\mathfrak{D}}\left(\mathfrak{C} \otimes_{A} B, \mathfrak{D}\right) \simeq B \otimes_{A} \mathfrak{C}$.

Moreover, if $\mathfrak{C}$ and $\mathfrak{D}$ are coseparable, then the condition "injector" in (b) can be deleted.

Proof. First observe that $-\otimes_{A} B$ is a Frobenius functor if and only if $\left(-\otimes_{A} B,-\square_{\mathfrak{D}}\left(B \otimes_{A} \mathfrak{C}\right)\right)$ is a Frobenius pair (by [18, Proposition 5.4]). A straightforward computation shows that the map $\rho_{M} \otimes_{A} B: M \otimes_{A} B \rightarrow M \otimes_{A} \mathfrak{C} \otimes_{A} B$ is a homomorphism of $\mathfrak{D}$-comodules. We have thus a commutative diagram in $\mathcal{M}^{\mathfrak{C}}$ with exact row

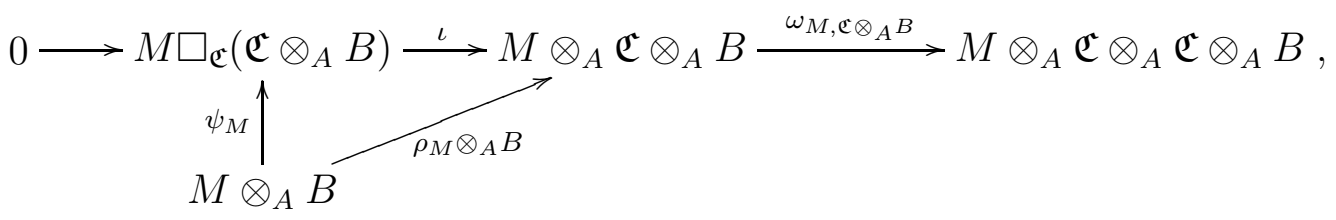

where $\psi_{M}$ is defined by the universal property of the kernel. Since ${ }_{B} \mathfrak{D}$ is flat, to prove that $\psi_{M}$ is an isomorphism of $\mathfrak{D}$-comodules it is enough to check that it is bijective, as the forgetful functor $U: \mathcal{M}^{\mathcal{D}} \rightarrow \mathcal{M}_{B}$ is faithfully exact. Some easy computations show that the map $\left(M \otimes_{A} \epsilon_{\mathfrak{C}} \otimes_{A} B\right) \circ \iota$ is the inverse in $\mathcal{M}_{B}$ to $\psi_{M}$. From this, we deduce a natural isomorphism $\psi:-\otimes_{A} B \simeq-\square_{\mathfrak{C}}\left(\mathfrak{C} \otimes_{A} B\right)$. The equivalence between (a) and (b) is then obvious from Proposition 2.9 and Proposition 2.7

When applied to the case where $\mathfrak{C}=A$ and $\mathfrak{D}=B$ are the trivial corings (which are separable), Theorem 4.1 gives functorial Morita's characterization of Frobenius ring extensions given in [27, Theorem 5.1]. This follows from [18, Example 4.3]: In the case $\mathfrak{C}=A, \mathfrak{D}=B$ we have that $A \otimes_{A} B \cong B$ is quasi-finite as a right $B$-comodule if and only if ${ }_{A} B$ is finitely generated an projective, and, in this case, $\mathrm{h}_{B}(B,-) \simeq-\otimes_{B} \operatorname{Hom}_{A}\left({ }_{A} B, A\right)$.

Theorem 4.3 generalizes the characterization of Frobenius extension of coalgebras over fields [13, Theorem 3.5]. It is then reasonable to give the following definition.

Definition 4.2. Let $(\varphi, \rho): \mathfrak{C} \rightarrow \mathfrak{D}$ be a homomorphism of corings such that ${ }_{A} \mathfrak{C}$ and ${ }_{B} \mathfrak{D}$ are flat. It is said to be a right Frobenius morphism of corings if $-\otimes_{A} B: \mathcal{M}^{\mathfrak{C}} \rightarrow \mathcal{M}^{\mathfrak{D}}$ is a Frobenius functor.

Theorem 4.3. Suppose that the algebras $A$ and $B$ are $Q F$ rings.

Let $(\varphi, \rho): \mathfrak{C} \rightarrow \mathfrak{D}$ be a homomorphism of corings such that the modules ${ }_{A} \mathfrak{C},{ }_{B} \mathfrak{D}$ and $\mathfrak{D}_{B}$ are projective. Then the following statements are equivalent

(a) $-\otimes_{A} B: \mathcal{M}^{\mathfrak{C}} \rightarrow \mathcal{M}^{\mathfrak{D}}$ is a Frobenius functor;

(b) the $\mathfrak{C}-\mathfrak{D}$-bicomodule $\mathfrak{C} \otimes_{A} B$ is quasi-finite as a right $\mathfrak{D}$-comodule, $\left(\mathfrak{C} \otimes_{A} B\right)_{\mathfrak{D}}$ is injective and there exists an isomorphism of $\mathfrak{D}-\mathfrak{C}$-bicomodules $\mathrm{h}_{\mathfrak{D}}\left(\mathfrak{C} \otimes_{A} B, \mathfrak{D}\right) \simeq B \otimes_{A} \mathfrak{C}$. 
Proof. Obvious from Proposition 3.10.

Now, suppose that the forgetful functor $\mathcal{M}^{\mathfrak{E}} \rightarrow \mathcal{M}_{A}$ is a Frobenius functor. Then the functor $-\otimes_{A} \mathfrak{C}: \mathcal{M}_{A} \rightarrow \mathcal{M}_{A}$ is also a Frobenius functor (since it is a composition of two Frobenius functors) and ${ }_{A} \mathfrak{C}$ is finitely generated projective. On the other hand, since $-\otimes_{A} \mathfrak{C}: \mathcal{M}_{A} \rightarrow \mathcal{M}^{\mathfrak{C}}$ is a left adjoint to $\operatorname{Hom}_{\mathfrak{C}}(\mathfrak{C},-): \mathcal{M}^{\mathfrak{C}} \rightarrow \mathcal{M}_{A}$. Then $\operatorname{Hom}_{\mathfrak{C}}(\mathfrak{C},-)$ is a Frobenius functor. Therefore, $\mathfrak{C}$ is finitely generated projective in $\mathcal{M}^{\mathfrak{C}}$, and hence in $\mathcal{M}_{A}$.

Lemma 4.4. Let $R$ be the opposite algebra of ${ }^{*} \mathfrak{C}$.

(1) $\mathfrak{C} \in \mathfrak{C}^{A}$ is quasi-finite (resp. quasi-finite and injector) as a right $A$-comodule if and only if ${ }_{A} \mathfrak{C}$ is finitely generated projective (resp. ${ }_{A} \mathfrak{C}$ is finitely generated projective and ${ }_{A} R$ is flat $)$. Let $\mathrm{h}_{A}(\mathfrak{C},-)=-\otimes_{A} R: \mathcal{M}^{A} \rightarrow \mathcal{M}^{\mathfrak{C}}$ be the cohom functor.

(2) If ${ }_{A} \mathfrak{C}$ is finitely generated projective and ${ }_{A} R$ is flat, then

$$
{ }_{A} \mathrm{~h}_{A}(\mathfrak{C}, A)_{\mathfrak{C}} \simeq{ }_{A} R_{\mathfrak{C}},
$$

where the right $\mathfrak{C}$-comodule structure of $R$ is defined as in [5, Lemma 4.3].

Proof. (1) Straightforward from [18, Example 4.3].

(2) From [5. Lemma 4.3], the forgetful functor $\mathcal{M}^{\mathfrak{c}} \rightarrow \mathcal{M}_{A}$ is the composition of functors $\mathcal{M}^{\mathfrak{C}} \rightarrow \mathcal{M}_{R} \rightarrow \mathcal{M}_{A}$. By [18, Proposition 4.2], $\mathrm{h}_{A}(\mathfrak{C},-)$ is a left adjoint to $-\square_{\mathfrak{C}} \mathfrak{C}: \mathcal{M}^{\mathfrak{C}} \rightarrow$ $\mathcal{M}_{A}$ which is isomorphic to the forgetful functor $\mathcal{M}^{\mathfrak{C}} \rightarrow \mathcal{M}_{A}$. Then $\mathrm{h}_{A}(\mathfrak{C},-)$ is isomorphic to the composition of functors

$$
\mathcal{M}_{A} \stackrel{-\otimes_{A} R}{\longrightarrow} \mathcal{M}_{R} \longrightarrow \mathcal{M}^{\mathfrak{C}} .
$$

In particular, ${ }_{A} \mathrm{~h}_{A}(\mathfrak{C}, A)_{\mathfrak{C}} \simeq{ }_{A}\left(A \otimes_{A} R\right)_{\mathfrak{C}} \simeq{ }_{A} R_{\mathfrak{C}}$.

Corollary 4.5. ([8, 27.10])

Let $\mathfrak{C}$ be an $A$-coring and let $R$ be the opposite algebra of ${ }^{*} \mathfrak{C}$. Then the following statements are equivalent

(a) The forgetful functor $F: \mathcal{M}^{\mathfrak{C}} \rightarrow \mathcal{M}_{A}$ is a Frobenius functor;

(b) ${ }_{A} \mathfrak{C}$ is finitely generated projective and $\mathfrak{C} \simeq R$ as $(A, R)$-bimodules, where $\mathfrak{C}$ is a right $R$-module via $c . r=c_{(1)} \cdot r\left(c_{(2)}\right)$, for all $c \in \mathfrak{C}$ and $r \in R$.

Proof. Straightforward from Theorem 4.1 and Lemma 4.4.

The following proposition gives sufficient conditions to have that a morphism of corings is right Frobenius if and only if it is left Frobenius. Note that it says in particular that the notion of Frobenius homomorphism of coalgebras over fields (by (b)) or of rings (by (d)) is independent on the side. Of course, the latter is well-known.

Proposition 4.6. Let $(\varphi, \rho): \mathfrak{C} \rightarrow \mathfrak{D}$ be a homomorphism of corings such that ${ }_{A} \mathfrak{C},{ }_{B} \mathfrak{D}$, $\mathfrak{C}_{A}$ and $\mathfrak{D}_{B}$ are flat. Assume that at least one of the following holds 
(a) $\mathfrak{C}$ and $\mathfrak{D}$ have a duality, and ${ }_{A} B$ and $B_{A}$ are flat;

(b) $A$ and $B$ are von Neumann regular rings;

(c) $B \otimes_{A} \mathfrak{C}$ is coflat in ${ }^{\mathfrak{D}} \mathcal{M}$ and $\mathfrak{C} \otimes_{A} B$ is coflat in $\mathcal{M}^{\mathfrak{D}}$ and ${ }_{A} B$ and $B_{A}$ are flat;

(d) $\mathfrak{C}$ and $\mathfrak{D}$ are coseparable corings.

Then the following statements are equivalent

1. $-\otimes_{A} B: \mathcal{M}^{\mathfrak{C}} \rightarrow \mathcal{M}^{\mathfrak{D}}$ is a Frobenius functor;

2. $B \otimes_{A}-:{ }^{\mathfrak{C}} \mathcal{M} \rightarrow{ }^{\mathfrak{D}} \mathcal{M}$ is a Frobenius functor.

Proof. Obvious from Theorem 2.11 and Theorem 3.11

Let us finally show how to derive from our results a remarkable characterization of the so called Frobenius corings.

Corollary 4.7. ([8, 27.8])

The following statements are equivalent

(a) the forgetful functor $\mathcal{M}^{\mathfrak{C}} \rightarrow \mathcal{M}_{A}$ is a Frobenius functor;

(b) the forgetful functor ${ }^{\mathfrak{C}} \mathcal{M} \rightarrow{ }_{A} \mathcal{M}$ is a Frobenius functor;

(c) there exist an $(A, A)$-bimodule map $\eta: A \rightarrow \mathfrak{C}$ and a $(\mathfrak{C}, \mathfrak{C})$-bicomodule map $\pi: \mathfrak{C} \otimes_{A}$ $\mathfrak{C} \rightarrow \mathfrak{C}$ such that $\pi\left(\mathfrak{C} \otimes_{A} \eta\right)=\mathfrak{C}=\pi\left(\eta \otimes_{A} \mathfrak{C}\right)$.

Proof. The proof of "(1) $\Leftrightarrow(4)$ " in Proposition 2.7 for $X=\mathfrak{C} \in{ }^{A} \mathcal{M}^{\mathfrak{C}}$ and $\Lambda=\mathfrak{C} \in{ }^{\mathfrak{c}} \mathcal{M}^{A}$ remains valid for our situation. Finally, notice that the condition (4) in this case is exactly the condition $(\mathrm{c})$.

\section{Applications to entwined modules}

In this section we particularize some our results in Section 4 to the category of entwined modules. We adopt the notations of [12]. We start with some remarks.

(1) Consider a right-right entwining structure $(A, C, \psi) \in \mathbb{E}_{\bullet}^{\bullet}(k)$ and a left-left entwining structure $(B, D, \varphi) \in: \mathbb{E}(k)$. The category of two-sided entwined modules ${ }_{B}^{D} \mathcal{M}(\varphi, \psi)_{A}^{C}$ defined in [12, pp. 68-69] is isomorphic to the category of bicomodules ${ }^{D \otimes B} \mathcal{M}^{A \otimes C}$ over the associated corings.

(2) If $(A, C, \psi)$ and $\left(A^{\prime}, C^{\prime}, \psi^{\prime}\right)$ belong to $\mathbb{E}_{\bullet}^{\bullet}(k)$ and are such that $\psi$ is an isomorphism, then $\psi$ is an isomorphism of corings (see [12, Proposition 34]), and consequently if the coalgebra $C$ is flat as a $k$-module, then the modules ${ }_{A}(A \otimes C)$ and $(A \otimes C)_{A}$ are flat, and

$$
{ }^{A \otimes C} \mathcal{M}^{A^{\prime} \otimes C^{\prime}} \simeq{ }_{A}^{C} \mathcal{M}\left(\psi^{-1}, \psi^{\prime}\right)_{A^{\prime}}^{C^{\prime}}
$$


(3) Let $(\alpha, \gamma):(A, C, \psi) \rightarrow\left(A^{\prime}, C^{\prime}, \psi^{\prime}\right)$ be a morphism in $\mathbb{E}:(k)$. We know that $(\alpha \otimes \gamma, \alpha)$ : $A \otimes C \rightarrow A^{\prime} \otimes C^{\prime}$ is a morphism of corings. The functor $F$ defined in [12, Lemma 8] satisfies the commutativity of the diagram

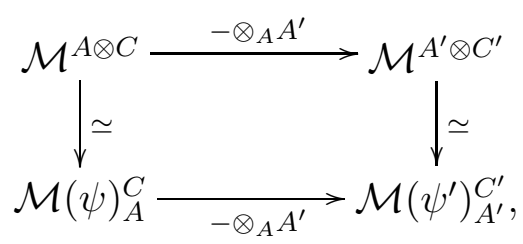

where $-\otimes_{A} A^{\prime}: \mathcal{M}^{A \otimes C} \rightarrow \mathcal{M}^{A^{\prime} \otimes C^{\prime}}$ is the induction functor defined in 18, Proposition $5.3]$.

We obtain the following result concerning the category of entwined modules.

Theorem 5.1. Let $(\alpha, \gamma):(A, C, \psi) \rightarrow\left(A^{\prime}, C^{\prime}, \psi^{\prime}\right)$ be a morphism in $\mathbb{E}:(k)$, such that ${ }_{k} C$ and ${ }_{k} D$ are flat.

1. The following statements are equivalent

(a) The functor $F=-\otimes_{A} A^{\prime}: \mathcal{M}(\psi)_{A}^{C} \rightarrow \mathcal{M}\left(\psi^{\prime}\right)_{A^{\prime}}^{C^{\prime}}$ defined in [12, Lemma 8] is a Frobenius functor;

(b) the $A \otimes C-A^{\prime} \otimes C^{\prime}$-bicomodule $(A \otimes C) \otimes_{A} A^{\prime}$ is quasi-finite injector as a right $A^{\prime} \otimes C^{\prime}$-comodule and there exists an isomorphism of $A^{\prime} \otimes C^{\prime}-A \otimes C$-bicomodules $\mathrm{h}_{A^{\prime} \otimes C^{\prime}}\left((A \otimes C) \otimes_{A} A^{\prime}, A^{\prime} \otimes C^{\prime}\right) \simeq A^{\prime} \otimes_{A}(A \otimes C)$.

Moreover, if $A \otimes C$ and $A^{\prime} \otimes C^{\prime}$ are coseparable corings, then the condition "injector" in (b) can be deleted.

2. If $A$ and $A^{\prime}$ are $Q F$ rings and the module $\left(A^{\prime} \otimes C^{\prime}\right)_{A^{\prime}}$ is projective, then the following are equivalent

(a) The functor $F=-\otimes_{A} A^{\prime}: \mathcal{M}(\psi)_{A}^{C} \rightarrow \mathcal{M}\left(\psi^{\prime}\right)_{A^{\prime}}^{C^{\prime}}$ defined in [12, Lemma 8] is a Frobenius functor;

(b) the $A \otimes C-A^{\prime} \otimes C^{\prime}$-bicomodule $(A \otimes C) \otimes_{A} A^{\prime}$ is quasi-finite and injective as a right $A^{\prime} \otimes C^{\prime}$-comodule and there exists an isomorphism of $A^{\prime} \otimes C^{\prime}-A \otimes C$-bicomodules $\mathrm{h}_{A^{\prime} \otimes C^{\prime}}\left((A \otimes C) \otimes_{A} A^{\prime}, A^{\prime} \otimes C^{\prime}\right) \simeq A^{\prime} \otimes_{A}(A \otimes C)$.

Proof. Follows from Theorem 4.1 and Theorem 4.3.

Remark 5.2. Let a right-right entwining structure $(A, C, \psi) \in \mathbb{E}:(k)$. The coseparability of the coring $A \otimes C$ is characterized in [12, Theorem 38(1)] (see also [5, Corollary 3.6]). 


\section{Applications to graded ring theory}

In this section we apply our results in the previous sections to the category of graded modules by a $G$-set. Let $G$ be a group, $A$ be a $G$-graded $k$-algebra, and let $X$ be a left $G$-set. The category $(G, X, A)-g r$ of graded left modules by $X$ is introduced and studied in [29]. A study of the graded ring theory can be found in the recent book [30]. We adopt the notations of [32] and [12, and we begin by giving some useful lemmas.

\subsection{Some useful lemmas}

Let $C=k X$ and $C^{\prime}=k X^{\prime}$ be two grouplike coalgebras, where $X$ and $X^{\prime}$ are arbitrary sets. We know (see [12, Example 4]) that the category $\mathcal{M}^{C}$ is isomorphic to the category of $X$-graded modules. Moreover we have the following:

Lemma 6.1. For a $k$-module $M$ which is both a $X$-graded and a $X^{\prime}$-graded module, the following are equivalent

(a) $M \in C^{\prime} \mathcal{M}^{C}$;

(b) for every $m \in M, x \in X, x^{\prime} \in X^{\prime}, \quad{ }_{x^{\prime}}\left(m_{x}\right) \in M_{x}$;

(c) for every $m \in M, x \in X, x^{\prime} \in X^{\prime}, \quad\left({ }_{x^{\prime}} m\right)_{x} \in{ }_{x^{\prime}} M$;

(d) for every $m \in M, x \in X, x^{\prime} \in X^{\prime}, \quad{ }_{x^{\prime}}\left(m_{x}\right)=\left({ }_{x^{\prime}} m\right)_{x}$.

Proof. Let $M=\bigoplus_{x \in X} M_{x}=\bigoplus_{x^{\prime} \in X^{\prime} x^{\prime}} M$. At first observe that the condition $(a)$ is equivalent to the fact that the diagram

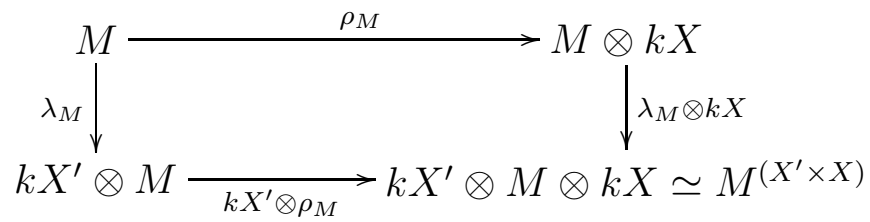

is commutative.

$(a) \Rightarrow(d)$ Let $m \in M$. We have, $\rho_{M}(m)=\sum_{x \in X} m_{x} \otimes x$ and $\lambda_{M}(m)=\sum_{x^{\prime} \in X^{\prime}} x^{\prime} \otimes_{x^{\prime}} m$. From the commutativity of the above diagram,

$$
\sum_{x \in X} \sum_{x^{\prime} \in X^{\prime}} x^{\prime} \otimes{ }_{x^{\prime}}\left(m_{x}\right) \otimes x=\sum_{x^{\prime} \in X^{\prime}} \sum_{x \in X} x^{\prime} \otimes\left({ }_{x}^{\prime} m\right)_{x} \otimes x .
$$

Hence, ${ }_{x^{\prime}}\left(m_{x}\right)=\left({ }_{x^{\prime}} m\right)_{x}$ for all $x \in X$, and $x^{\prime} \in X^{\prime}$.

$(d) \Rightarrow(b)$ Trivial.

$(b) \Rightarrow(a)$ Let $m \in M$. We have,

$$
\left(\lambda_{M} \otimes k X\right) \rho_{M}(m)=\sum_{x \in X} \sum_{x^{\prime} \in X^{\prime}} x^{\prime} \otimes_{x^{\prime}}\left(m_{x}\right) \otimes x
$$


and

$$
\left(k X^{\prime} \otimes \rho_{M}\right) \lambda_{M}(m)=\left(k X^{\prime} \otimes \rho_{M}\right)\left(\sum_{x \in X} \sum_{x^{\prime} \in X^{\prime}} x^{\prime} \otimes_{x^{\prime}}\left(m_{x}\right)\right)=\sum_{x \in X} \sum_{x^{\prime} \in X^{\prime}} x^{\prime} \otimes_{x^{\prime}}\left(m_{x}\right) \otimes x .
$$

Hence $(a)$ follows.

$(a) \Leftrightarrow(d) \Leftrightarrow(c)$ Follows by symmetry.

Now let $G$ and $G^{\prime}$ be two groups, $A$ be a $G$-graded $k$-algebra, $A^{\prime}$ be a $G^{\prime}$-graded $k$ algebra, $X$ be a right $G$-set, and $X^{\prime}$ be a left $G^{\prime}$-set. Let $k G$ and $k G^{\prime}$ be the canonical Hopf algebras.

Let $\psi: k X \otimes A \rightarrow A \otimes k X$, be the map defined by $\left[x \otimes a_{g} \mapsto a_{g} \otimes x g\right]$, and $\psi^{\prime}$ : $A^{\prime} \otimes k X^{\prime} \rightarrow k X^{\prime} \otimes A^{\prime}$, be the map defined by $\left[a_{g^{\prime}}^{\prime} \otimes x^{\prime} \mapsto g^{\prime} x^{\prime} \otimes a_{g^{\prime}}^{\prime}\right]$.

From [12, §4.6], we have $(k G, A, k X) \in \mathbb{D K}_{\bullet}^{\bullet}(k),\left(k G^{\prime}, A^{\prime}, k X^{\prime}\right) \in \bullet \mathbb{D} \mathbb{K}(k),(A, k X, \psi) \in$ $\mathbb{E}_{\bullet}^{\bullet}(k),\left(A^{\prime}, k X^{\prime}, \psi^{\prime}\right) \in \bullet \mathbb{E}(k)$, and $\mathcal{M}(k G)_{A}^{k X} \simeq g r-(A, X, G),{ }_{A^{\prime}}^{k X^{\prime}} \mathcal{M}\left(k G^{\prime}\right) \simeq\left(G^{\prime}, X^{\prime}, A^{\prime}\right)-$ $g r$.

Lemma 6.2. (1) Let $M$ be a $k$-module having the structure of $X$-graded right $A$-module and $X^{\prime}$-graded left $A^{\prime}$-module. The following are equivalent

(a) $M \in{ }_{A^{\prime}}^{k X^{\prime}} \mathcal{M}\left(\psi^{\prime}, \psi\right)_{A}^{k X}$;

(b) the following conditions hold

(i) $M$ is a $\left(A^{\prime}, A\right)$-bimodule,

(ii) for every $m \in M, x \in X, x^{\prime} \in X^{\prime}, \quad{ }_{x^{\prime}}\left(m_{x}\right) \in M_{x}$ (or $\left({ }_{x^{\prime}} m\right)_{x} \in{ }_{x^{\prime}} M$, or $\left.{ }_{x^{\prime}}\left(m_{x}\right)=\left({ }_{x^{\prime}} m\right)_{x}\right)$,

(iii) for every $x \in X, M_{x}$ is a submodule of $A_{A^{\prime}} M$,

(iv) for every $x^{\prime} \in X^{\prime},{ }_{x^{\prime}} M$ is a submodule of $M_{A}$.

(2) $M \in{ }_{A^{\prime}}^{k X^{\prime}} \mathcal{M}\left(\psi^{\prime}, \psi\right)_{A}^{k X}$ if and only if $M$ is an $X^{\prime} \times X$-graded $A^{\prime}-A$-bimodule (see [32, pp. 492-493]).

Proof. (1) $(a) \Leftrightarrow(b)$ We will use the definition of an object in the category of two-sided entwined modules ${ }_{A^{\prime}}^{k X^{\prime}} \mathcal{M}\left(\psi^{\prime}, \psi\right)_{A}^{k X}$ (see [12, pp. 68-69]). By Lemma 6.1, the condition " $M \in{ }^{k X^{\prime}} \mathcal{M}^{k X "}$ is equivalent to the condition $(i i)$. We have moreover that the left $A^{\prime}$ action on $M$ is $k X$-colinear if and only if for every $x \in X, m \in M_{x}, \rho_{M}\left(a^{\prime} m\right)=\left(a^{\prime} m\right) \otimes x$, if and only if for every $x \in X, m \in M_{x}, a^{\prime} m \in M_{x}$, if and only if (iii) holds. By symmetry, the condition "the right $A$-action on $M$ is $k X^{\prime}$-colinear" is equivalent to the condition $(i v)$.

(2) The "if" part is clear (see [32, p. 493]). For the "only if" part, put $M=$ $\bigoplus_{\left(x^{\prime}, x\right) \in X^{\prime} \times X} M_{\left(x^{\prime}, x\right)}$, where $\left.M_{\left(x^{\prime}, x\right)}={ }_{x^{\prime}}\left(M_{x}\right)={ }_{{ }_{x^{\prime}}} M\right)_{x}$. Let $a^{\prime} \in A_{g^{\prime}}^{\prime}, m \in M$. Since $a^{\prime} \cdot x^{\prime}\left(m_{x}\right) \in M_{x}$ and $a^{\prime} \cdot x^{\prime}\left(m_{x}\right) \in g^{\prime} x^{\prime}(M), a^{\prime} \cdot x^{\prime}\left(m_{x}\right) \in a^{\prime} x^{\prime}\left(M_{x}\right)$. Therefore, $A_{g^{\prime} \cdot x^{\prime}}^{\prime}\left(M_{x}\right) \subset$ $a^{\prime} x^{\prime}\left(M_{x}\right)$. By symmetry we obtain $A_{g^{\prime}}^{\prime} M_{\left(x^{\prime}, x\right)} A_{g} \subset M_{g^{\prime}\left(x^{\prime}, x\right) g}=M_{\left(g^{\prime} x^{\prime}, x g\right)}\left(g \in G, g^{\prime} \in G^{\prime}, x \in\right.$ $\left.X, x^{\prime} \in X^{\prime}\right)$. 


\subsection{Adjoint pairs and Frobenius pairs of functors between cate- gories of graded modules over $G$-sets}

Throughout this subsection, $G$ and $G^{\prime}$ will be two groups, $A$ will be a $G$-graded $k$-algebra, $A^{\prime}$ will be a $G^{\prime}$-graded $k$-algebra, $X$ will be a right $G$-set, and $X^{\prime}$ will be a right $G^{\prime}$-set. Let $k G$ and $k G^{\prime}$ be the canonical Hopf algebras. Let $\psi: k X \otimes A \rightarrow A \otimes k X$ be the map defined by $\left[x \otimes a_{g} \mapsto a_{g} \otimes x g\right]$. Analogously we define the map $\psi^{\prime}: k X^{\prime} \otimes A^{\prime} \rightarrow A^{\prime} \otimes k X^{\prime}$. The coaction and the counit of the coring $A \otimes k X$ are defined by:

$$
\Delta_{A \otimes k X}(a \otimes x)=(a \otimes x) \otimes_{A}\left(1_{A} \otimes x\right), \quad \epsilon_{A \otimes k X}(a \otimes x)=a \quad(a \in A, x \in X) .
$$

An important result is that the corings $A \otimes k X$ and $A^{\prime} \otimes k X^{\prime}$ are coseparable. A proof is clear by using [5, Corollary 3.6] and [12, Proposition 101]. A direct proof in the setting of corings is deduced from [5, Theorem 3.5] by using the cointegral in the coring $A \otimes k X$ given by $\delta(a \otimes x \otimes y)=a \delta_{x, y}$ (Kronecker's delta) for $a \in A, x, y \in X$ (see [8, 26.2] for the definition of a cointegral in a coseparable coring).

Lemma 6.3. (1) $\psi$ is bijective, $\left(A, k X, \psi^{-1}\right) \in \bullet_{\bullet} \mathbb{E}(k)$, and

$$
{ }_{A}^{k X} \mathcal{M}(k G):={ }_{A}^{k X} \mathcal{M}\left(\psi^{-1}\right) \simeq(G, X, A)-g r
$$

where the structure of left $G$-set on $X$ is given by $g . x=x g^{-1}(g \in G, x \in X)$.

(2) Every object of the category ${ }^{A^{\prime} \otimes k X^{\prime}} \mathcal{M}^{A \otimes k X} \simeq{ }_{A^{\prime}}^{X^{\prime}} \mathcal{M}\left(\left(\psi^{\prime}\right)^{-1}, \psi\right)_{A}^{k X}$ can be identified to an $X^{\prime} \times X$-graded $A^{\prime}-A$-bimodule.

Proof. (1) From [12, Proposition 2], and since the Hopf algebra $H=k G$ is cocommutative, $S \circ S=1_{H}$ and $\bar{S}=S^{-1}=S$ is a twisted antipode of $H$. Hence $\psi$ is bijective and $\left(A, k X, \psi^{-1}\right) \in: \mathbb{E}(k)$ (see [12, p. 49]), and ${ }_{A}^{k X} \mathcal{M}(k G):={ }_{A}^{k X} \mathcal{M}\left(\psi^{-1}\right) \simeq(G, X, A)-g r$, since $\psi^{-1}: A \otimes k X \rightarrow k X \otimes A,\left[a_{g} \otimes x \mapsto g . x \otimes a_{g}\right]$, where $g . x=x g^{-1}$.

(2) It follows from (1) and Lemma 6.2.

Lemma 6.4. (1) Let $M \in g r-(A, X, G)$, and $N \in(G, X, A)-g r$. We know that $M \in$ $\mathcal{M}^{A \otimes k X}$ by the coaction: $\rho_{M}: M \rightarrow M \otimes_{A}(A \otimes k X)$, where $\rho_{M}\left(m_{x}\right)=m_{x} \otimes_{A}\left(1_{A} \otimes x\right)$, and $N \in{ }^{A \otimes k X} \mathcal{M}$ by the coaction: $\lambda_{N}: N \rightarrow(A \otimes k X) \otimes_{A} N$, where $\lambda_{N}\left({ }_{x} n\right)=$ $\left(1_{A} \otimes x\right) \otimes_{A} n$. We have $M \square_{(A \otimes k X)} N=M \widehat{\otimes}_{A} N$, where $M \widehat{\otimes}_{A} N$ is the additive subgroup of $M \otimes_{A} N$ generated by the elements $m \otimes_{A} n$ where $x \in X, m \in M_{x}$, and $n \in{ }_{x} N$ (see [32, p. 492]).

(2) Let $P$ be an $X \times X^{\prime}$-graded $A-A^{\prime}$-bimodule. We have the commutative diagram:

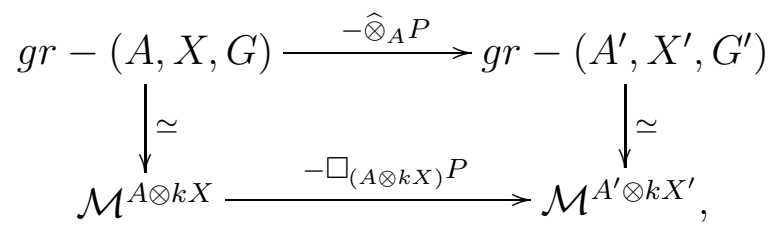

where $-\widehat{\otimes}_{A} P: g r-(A, X, G) \rightarrow g r-\left(A^{\prime}, X^{\prime}, G^{\prime}\right)$ is the functor defined in [32, $\left.p .493\right]$. 
Proof. (1) At first we will show that the right $A$-module $A \otimes k X$ is free. More precisely that the family $\left\{1_{A} \otimes x \mid x \in X\right\}$ is a right basis of it. It is clear that $a_{g} \otimes x=\left(1_{A} \otimes x g^{-1}\right) a_{g}$ for $a_{g} \in A_{g}$ and $x \in X$. Now suppose that $\sum_{i}\left(1_{A} \otimes x_{i}\right) a_{i}=0$, where $x_{i} \in X, a_{i} \in A$. Then $\sum_{i} \psi\left(x_{i} \otimes a_{i}\right)=0$. Since $\psi$ is bijective, we obtain $\sum_{i} x_{i} \otimes a_{i}=0$. Therefore $a_{i}=0$ for all $i$. Hence the above mentioned family is a basis of the right $A$-module $A \otimes k X$. (There is a shorter and indirect proof of this fact by using that $\psi$ is an isomorphism of $A$-bimodules, and $\left\{x \otimes 1_{A} \mid x \in X\right\}$ is a basis of the right $A$-module $\left.k X \otimes A\right)$.

Finally, suppose that $\sum_{i} m_{x_{i}} \otimes_{A y_{i}} n \in M \square_{(A \otimes k X)} N$. Then

$$
\sum_{i} m_{x_{i}} \otimes_{A} 1_{A} \otimes x_{i} \otimes_{A y_{i}} n=\sum_{i} m_{x_{i}} \otimes_{A} 1_{A} \otimes y_{i} \otimes_{A y_{i}} n
$$

Hence (the right $A$-module $A \otimes k X$ is free), $x_{i}=y_{i}$ for all $i$, and $M \square_{(A \otimes k X)} N \subset M \widehat{\otimes}_{A} N$. The other inclusion is obvious.

(2) It suffices to show that the map $M \widehat{\otimes}_{A} P \rightarrow M \widehat{\otimes}_{A} P \otimes_{A^{\prime}}\left(A^{\prime} \otimes k X^{\prime}\right)$ defined by

$$
\left[\sum_{x \in F} m_{x} \otimes_{A x} p \longmapsto \sum_{x^{\prime} \in X^{\prime}}\left(\sum_{x \in F} m_{x} \otimes_{A x} p\right)_{x^{\prime}} \otimes_{A^{\prime}}\left(1_{A^{\prime}} \otimes x^{\prime}\right)\right],
$$

where $F$ is a finite subset of $X$, makes commutative the following diagram

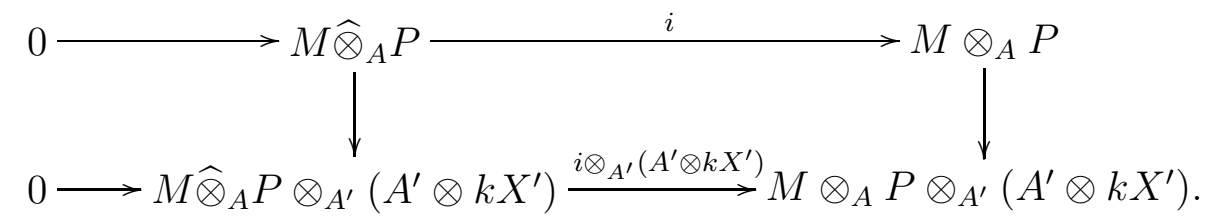

That is clear since $\left(\sum_{x \in F} m_{x} \otimes_{A x} p\right)_{x^{\prime}}=\sum_{x \in F} m_{x} \otimes_{A}\left({ }_{x} p\right)_{x^{\prime}}=\sum_{x \in F} m_{x} \otimes_{A} p_{\left(x, x^{\prime}\right)}$, where $p=\sum_{x \in F} p$.

Let $\widehat{A}=A \otimes k X$ be the $X \times X$-graded $A-A$-bimodule associated to the $(A \otimes k X)-$ $(A \otimes k X)$ - bicomodule $A \otimes k X$. It is clear that $-\widehat{\otimes}_{A} \widehat{A}=-\square_{(A \otimes k X)}(A \otimes k X) \simeq 1_{g r-(A, G, X)}$. The gradings are $\widehat{A}_{x}=A \otimes k x$, and ${ }_{x} \widehat{A}=\left\{\sum_{i} a_{i} \otimes x_{i} \mid x_{i} g^{-1}=x, \forall i, \forall g \in G:\left(a_{i}\right)_{g} \neq 0\right\}$ $(x \in X)$. Recall from [24, Proposition 1.2] that for every $X \times X^{\prime}$-graded $A-A^{\prime}$-bimodule $P$, we have an adjunction $\left(-\widehat{\otimes}_{A} P, \mathrm{H}\left(P_{A^{\prime}},-\right)\right)$. The unit and the counit of this adjunction are given respectively by $\eta_{M}: M \rightarrow \mathrm{H}\left(P_{A^{\prime}}, M \widehat{\otimes}_{A} P\right), \eta_{M}(m)(p)=\sum_{x \in X} m_{x} \otimes_{A x} p(m=$ $\left.\sum_{x \in X} m_{x} \in M, p=\sum_{x \in X} x \in P\right)$, and $\varepsilon_{N}: \mathrm{H}\left(P_{A^{\prime}}, N\right) \widehat{\otimes}_{A} P \rightarrow N, \varepsilon_{N}\left(f \otimes_{A} P\right)=f(p)$ $\left(f \in \mathrm{H}\left(P_{A^{\prime}}, N\right)_{x}, p \in{ }_{x} P, x \in X\right)$.

Proposition 6.5. (24, Proposition 1.3, Corollary 1.4])

(1) The following statements are equivalent for a k-linear functor $F: g r-(A, X, G) \rightarrow$ $g r-\left(A^{\prime}, X^{\prime}, G^{\prime}\right)$.

(a) F has a right adjoint; 
(b) $F$ is right exact and preserves coproducts;

(c) $F \simeq-\widehat{\otimes}_{A} P$ for some $X \times X^{\prime}$-graded $A-A^{\prime}$-bimodule $P$.

(2) A k-linear functor $G: g r-\left(A^{\prime}, X^{\prime}, G^{\prime}\right) \rightarrow g r-(A, X, G)$ has a left adjoint if and only if $G \simeq \mathrm{H}\left(P_{A^{\prime}},-\right)$ for some $X \times X^{\prime}$-graded $A-A^{\prime}$-bimodule $P$.

Proof. (1) $(a) \Rightarrow(b)$ Clear. $(b) \Rightarrow(c)$ It follows from Theorem 2.3 and Lemma 6.4. $(c) \Rightarrow(a)$ Obvious from the above mentioned result.

(2) It follows from (1) and [24, Proposition 1.2].

Lemma 6.6. Let $P$ be an $X \times X^{\prime}$-graded $A-A^{\prime}$-bimodule.

(1) $\mathrm{H}\left(P_{A^{\prime}},-\right)$ is right exact and preserves direct limits if and only if ${ }_{x} P$ is finitely generated projective in $\mathcal{M}_{A^{\prime}}$ for every $x \in X$.

(2) Suppose that ${ }_{x} P$ is finitely generated projective in $\mathcal{M}_{A^{\prime}}$ for every $x \in X$.

(a) For every $k$-algebra $T$,

$$
\Upsilon_{Z, M}: Z \otimes_{T} \mathrm{H}\left(P_{A^{\prime}}, M\right) \stackrel{\simeq}{\longrightarrow} \mathrm{H}\left(P_{A^{\prime}}, Z \otimes_{T} M\right)
$$

defined by

$$
\Upsilon_{Z, M}\left(z \otimes_{T} f\right)(p)=\sum_{x \in X} z \otimes_{T} f_{x}\left({ }_{x} p\right)
$$

$\left(z \in Z, f=\sum_{x \in X} f_{x} \in \mathrm{H}\left(P_{A^{\prime}}, M\right), p=\sum_{x \in X} x p \in P\right)$, is the natural isomorphism associated to the functor (see Section 国)

$$
\mathrm{H}\left(P_{A^{\prime}},-\right): g r-\left(A^{\prime}, X^{\prime}, G^{\prime}\right) \rightarrow g r-(A, X, G) .
$$

(b) Moreover we have the natural isomorphism

$$
\eta_{N}: N \widehat{\otimes}_{A^{\prime}} \mathrm{H}\left(P_{A^{\prime}}, \widehat{A}^{\prime}\right) \stackrel{\simeq}{\longrightarrow} \mathrm{H}\left(P_{A^{\prime}}, N\right)
$$

defined by

$$
\eta_{N}\left(n_{x^{\prime}} \otimes_{A^{\prime} x^{\prime}} f\right)(p)=\sum_{x \in X} n_{x^{\prime}} \delta\left(\left(1_{A^{\prime}} \otimes x^{\prime}\right) \otimes_{A^{\prime}} f_{\left(x^{\prime}, x\right)}\left({ }_{x} p\right)\right)
$$

$\left(n_{x^{\prime}} \in N_{x^{\prime}},{ }_{x^{\prime}} f \in{ }_{x^{\prime}} \mathrm{H}\left(P_{A^{\prime}}, \widehat{A}^{\prime}\right), p=\sum_{x \in X} p \in P\right)$, where $\delta$ is the cointegral in the coring $A^{\prime} \otimes k X^{\prime}$ defined in the beginning of this subsection. The left grading on $\mathrm{H}\left(P_{A^{\prime}}, \widehat{A}^{\prime}\right)$ is given by

$$
\begin{aligned}
& { }_{x^{\prime}} \mathrm{H}\left(P_{A^{\prime}}, \widehat{A^{\prime}}\right)=\left\{f \in \mathrm{H}\left(P_{A^{\prime}}, \widehat{A^{\prime}}\right) \mid \Delta_{\left(A^{\prime} \otimes k X^{\prime}\right)}(f(p))=\left(1_{A^{\prime}} \otimes x^{\prime}\right) \otimes_{A^{\prime}} \sum_{x \in X} f_{x}(x p) \text { for all } p \in P\right\} \\
& \left(x^{\prime} \in X^{\prime}\right) .
\end{aligned}
$$


Proof. (1) We have

$$
\mathrm{H}\left(P_{A^{\prime}},-\right) \simeq \bigoplus_{x \in X} \operatorname{Hom}_{g r-\left(A^{\prime}, X^{\prime}, G^{\prime}\right)}\left({ }_{x} P,-\right): g r-\left(A^{\prime}, X^{\prime}, G^{\prime}\right) \longrightarrow \mathbf{A b} .
$$

Hence, $\mathrm{H}\left(P_{A^{\prime}},-\right)$ is right exact and preserves direct limits if and only if $\operatorname{Hom}_{g r-\left(A^{\prime}, X^{\prime}, G^{\prime}\right)}\left({ }_{x} P,-\right)$ is right exact and preserves direct limits for every $x \in X$ if and only if ${ }_{x} P$ is finitely generated projective in $\mathcal{M}_{A^{\prime}}$ for every $x \in X$ (by [33, Proposition V.3.4] and the fact that every finitely generated projective object of a Grothendieck category is finitely presented).

(2) (a) Let $T$ be a $k$-algebra. Let $M$ be an $X_{0} \times X^{\prime}$-graded $T-A^{\prime}$-bimodule, where $X_{0}$ is a singleton, $Z$ be a right $T$-module, and $x \in X$. We have a sequence of $T$-submodules

$$
\mathrm{H}\left(P_{A^{\prime}}, M\right)_{x} \leq \mathrm{H}\left(P_{A^{\prime}}, M\right) \leq \operatorname{Hom}_{g r-\left(A^{\prime}, X^{\prime}, G^{\prime}\right)}(P, M) \leq{ }_{T} \operatorname{Hom}_{A^{\prime}}\left(P_{A^{\prime}}, M\right),
$$

and the induced structure of left $T$-module on $\mathrm{H}\left(P_{A^{\prime}}, M\right)$ is the same structure (see Section 2) of left $T$-module associated to the functor $\mathrm{H}\left(P_{A^{\prime}},-\right)$ on it. Moreover, we have the isomorphism of left $T$-modules $\mathrm{H}\left(P_{A^{\prime}}, M\right)_{x} \simeq \operatorname{Hom}_{g r-\left(A^{\prime}, X^{\prime}, G^{\prime}\right)}\left({ }_{x} P, M\right)$. From 3 , Proposition 20.10], for every $x \in X$, there is an isomorphism

$$
\eta_{x}: Z \otimes_{T} \operatorname{Hom}_{A^{\prime}}\left({ }_{x} P, M\right) \stackrel{\simeq}{\longrightarrow} \operatorname{Hom}_{A^{\prime}}\left({ }_{x} P, Z \otimes_{T} M\right)
$$

defined by $\eta_{x}\left(z \otimes_{T} \gamma_{x}\right):{ }_{x} p \mapsto z \otimes_{T} \gamma_{x}\left({ }_{x} p\right)\left(z \in Z, \gamma_{x} \in \operatorname{Hom}_{A^{\prime}}\left({ }_{x} P, M\right)\right)$. For every $x \in X$, $\eta_{x}$ induces an isomorphism

$$
\eta_{x}^{\prime}: Z \otimes_{T} \operatorname{Hom}_{g r-\left(A^{\prime}, X^{\prime}, G^{\prime}\right)}\left({ }_{x} P, M\right) \stackrel{\simeq}{\longrightarrow} \operatorname{Hom}_{g r-\left(A^{\prime}, X^{\prime}, G^{\prime}\right)}\left({ }_{x} P, Z \otimes_{T} M\right) .
$$

Now let us consider the isomorphism

$$
\eta_{Z, M}^{\prime}:=\bigoplus_{x \in X} \eta_{x}^{\prime}: Z \otimes_{T} \mathrm{H}\left(P_{A^{\prime}}, M\right) \stackrel{\simeq}{\longrightarrow} \mathrm{H}\left(P_{A^{\prime}}, Z \otimes_{T} M\right)
$$

We have $\eta_{Z, M}^{\prime}\left(z \otimes_{T} f\right)(p)=\sum_{x \in X} z \otimes_{T} f_{x}\left({ }_{x} p\right)\left(z \in Z, f=\sum_{x \in X} f_{x} \in \mathrm{H}\left(P_{A^{\prime}}, M\right), p=\right.$ $\left.\sum_{x \in X}{ }_{x} p \in P\right)$. We can verify easily that $\eta_{Z, M}^{\prime}$ is a morphism of right $A$-modules. Hence it is a morphism in $g r-(A, X, G)$. It is clear that $\eta_{Z, M}^{\prime}$ is natural in $Z$, and $\eta_{T, M}^{\prime}$ makes commutative the diagram (11) (see Section 21). Finally, by Mitchell's Theorem [26, Theorem 3.6.5], $\eta_{Z, M}^{\prime}=\Upsilon_{Z, M}$.

(b) To prove the first statement it suffices to use the proof of Theorem 2.3, the property $(a)$, and the fact that every comodule over an $A$-coseparable coring is $A$-relative injective comodule (for the last see [8, 26.1]).

Finally, we will prove the last statement. We know that $\lambda_{\mathrm{H}\left(P_{A^{\prime}}, \widehat{A^{\prime}}\right)}$ is defined $([18])$ to be the unique $A^{\prime}$-linear map making commutative the following diagram

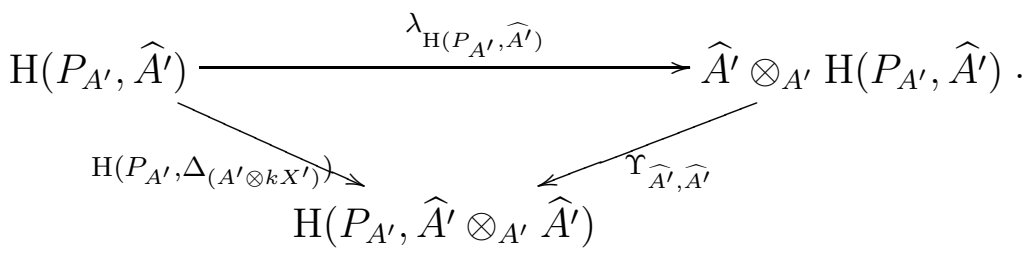


On the other hand, for each $x^{\prime} \in X^{\prime},{ }_{x^{\prime}} \mathrm{H}\left(P_{A^{\prime}}, \widehat{A}^{\prime}\right)=\left\{f \in \mathrm{H}\left(P_{A^{\prime}}, \widehat{A}^{\prime}\right) \mid \lambda_{\mathrm{H}\left(P_{A^{\prime}}, \widehat{A^{\prime}}\right)}(f)=\right.$ $\left.\left(1_{A^{\prime}} \otimes x^{\prime}\right) \otimes_{A^{\prime}} f\right\}$. Since $\Upsilon_{\widehat{A^{\prime}}, \widehat{A^{\prime}}}$ is an isomorphism, ${ }_{x^{\prime}} \mathrm{H}\left(P_{A^{\prime}}, \widehat{A}^{\prime}\right)=\left\{f \in \mathrm{H}\left(P_{A^{\prime}}, \widehat{A}^{\prime}\right)\right.$ $\Delta_{\left(A^{\prime} \otimes k X^{\prime}\right)}(f(p))=\left(1_{A^{\prime}} \otimes x^{\prime}\right) \otimes_{A^{\prime}} \sum_{x \in X} f_{x}\left({ }_{x} p\right)$ for all $\left.p \in P\right\}$.

Remark 6.7. Let $\Sigma$ be a finitely generated projective right $A$-module, and $M$ be a right $A$-module. It easy to verify that the map $\alpha_{\Sigma, M}: \operatorname{Hom}_{A}(\Sigma, M) \rightarrow M \otimes_{A} \Sigma^{*}$ defined by $\alpha_{\Sigma, M}(\varphi)=\sum_{i} \varphi\left(e_{i}\right) \otimes_{A} e_{i}^{*}$, where $\left\{e_{i}, e_{i}^{*}\right\}_{i}$ is a dual basis of $\Sigma$, is an isomorphism, with $\alpha_{\Sigma, M}^{-1}\left(m \otimes_{A} f\right)(u)=m f(u)\left(m \in M, f \in \Sigma^{*}, u \in \Sigma\right)$.

Now we assume that the condition of Lemma 6.6(2) holds. It is easy to verify that for every $x \in X, \eta_{x}=\alpha_{x P, Z \otimes_{T} M}^{-1} \circ\left(Z \otimes_{T} \alpha_{x} P, M\right)$, where $\eta_{x}$ is the isomorphism (5D). Therefore $\eta_{x}^{-1}=\left(Z \otimes_{T} \alpha_{x P, M}^{-1}\right) \circ \alpha_{x} P, Z \otimes_{T} M$. Hence $\Upsilon_{Z, M}^{-1}(g)=\sum_{x \in X} \sum_{i \in I_{x}}\left(Z \otimes_{T} \alpha_{x P, M}^{-1}\right)\left(g_{x}\left(e_{x, i}\right) \otimes_{A^{\prime}} e_{x, i}^{*}\right)$, where $\left\{e_{x, i}, e_{x, i}^{*}\right\}_{i \in I_{x}}$ is a dual basis of ${ }_{x} P(x \in X)$, and $g=\sum_{x \in X} g_{x} \in \mathrm{H}\left(P_{A^{\prime}}, Z \otimes_{T} M\right)$. Finally, for each $N \in g r-\left(A^{\prime}, X^{\prime}, G^{\prime}\right)$,

$$
\eta_{N}^{-1}: \mathrm{H}\left(P_{A^{\prime}}, N\right) \stackrel{\simeq}{\longrightarrow} N \widehat{\otimes}_{A^{\prime}} \mathrm{H}\left(P_{A^{\prime}}, \widehat{A}^{\prime}\right)
$$

is $\eta_{N}^{-1}=\Upsilon_{N, \widehat{A^{\prime}}}^{-1} \circ \mathrm{H}\left(P_{A^{\prime}}, \rho_{N}\right)$, and then

$$
\eta_{N}^{-1}(\varphi)=\sum_{x \in X} \sum_{i \in I_{x}} \sum_{x^{\prime} \in X^{\prime}}\left(\varphi_{x}\left(e_{x, i}\right)\right)_{x^{\prime}} \otimes_{A^{\prime}} \psi^{\prime}\left(x^{\prime} \otimes e_{x, i}^{*}(-)\right),
$$

where $\varphi=\sum_{x \in X} \varphi_{x} \in \mathrm{H}\left(P_{A^{\prime}}, N\right)$.

In particular, if $X^{\prime}=G^{\prime}=\left\{e^{\prime}\right\}$, then

$$
\eta_{N}^{-1}: \mathrm{H}\left(P_{A^{\prime}}, N\right) \stackrel{\simeq}{\longrightarrow} N \otimes_{A^{\prime}} \mathrm{H}\left(P_{A^{\prime}}, A^{\prime}\right)
$$

is defined by

$$
\eta_{N}^{-1}(\varphi)=\sum_{x \in X} \sum_{i \in I_{x}} \varphi\left(e_{x, i}\right) \otimes_{A^{\prime}} e_{x, i}^{*} \quad\left(\varphi \in \mathrm{H}\left(P_{A^{\prime}}, N\right)\right) .
$$

Lemma 6.8. (1) Let $N \in{ }_{A^{\prime}} \mathcal{M}^{A \otimes k X}$. Then $N$ is quasi-finite as a right $A \otimes k X$-comodule if and only if $N_{x}$ is finitely generated projective in $A^{\prime} \mathcal{M}$, for every $x \in X$. In this case, the cohom functor $\mathrm{h}_{A \otimes k X}(N,-)$ is the composite

$$
\mathcal{M}^{A \otimes k X} \stackrel{\simeq}{\longrightarrow} g r-(A, X, G) \stackrel{-\widehat{\otimes}_{A} P}{\longrightarrow} \mathcal{M}_{A^{\prime}}
$$

where $P$ is the $X \times X_{0}^{\prime}$-graded $A-A^{\prime}$-bimodule $\mathrm{H}\left({ }_{A^{\prime}} N, A^{\prime}\right)$ with $X_{0}^{\prime}$ is a singleton.

(2) Now suppose that $N \in{ }^{A^{\prime} \otimes k X^{\prime}} \mathcal{M}^{A \otimes k X}$, and $N_{x}$ is finitely generated projective in ${ }_{A^{\prime}} \mathcal{M}$, for every $x \in X$. Let $\left\{e_{x, i}, e_{x, i}^{*}\right\}_{i \in I_{x}}$ be a dual basis of $N_{x}(x \in X)$. Let $\theta: 1_{\mathcal{M}^{A \otimes k X}} \rightarrow$ $-\widehat{\otimes}_{A} P \otimes_{A^{\prime}} N$ be the unit of the adjunction $\left(-\widehat{\otimes}_{A} P,-\otimes_{A^{\prime}} N\right)$, and let $M \in g r-$ $(A, X, G)$. The coaction on $\mathrm{h}_{A \otimes k X}(N, M)=M \widehat{\otimes}_{A} P$ :

$$
\rho_{M \widehat{\otimes}_{A} P}: M \widehat{\otimes}_{A} P \rightarrow M \widehat{\otimes}_{A} P \otimes_{A^{\prime}}\left(A^{\prime} \otimes k X^{\prime}\right)
$$


is the unique $A^{\prime}$-linear map satisfying the condition:

$$
\sum_{x \in X} \sum_{i \in I_{x}} \rho_{M \widehat{\otimes}_{A} P}\left(m_{x} \otimes_{A} e_{x, i}^{*}\right) \otimes_{A^{\prime}} e_{x, i}=\sum_{x \in X} \sum_{i \in I_{x}} \sum_{x^{\prime} \in X^{\prime}} m_{x} \otimes_{A} e_{x, i}^{*} \otimes_{A^{\prime}}\left(1_{A^{\prime}} \otimes x^{\prime}\right) \otimes_{A^{\prime}}\left(e_{x, i}\right)_{x^{\prime}},
$$

for every $m \in M$.

Proof. (1) It follows from Lemma 6.4(2), that the functor $F:=-\widehat{\otimes}_{A^{\prime}} N: \mathcal{M}_{A^{\prime}}=g r-$ $\left(A^{\prime}, X_{0}^{\prime}, G^{\prime}\right) \rightarrow g r-(A, X, G)$, where $X_{0}^{\prime}$ is a singleton, is the composite

$$
\mathcal{M}_{A^{\prime}}=g r-\left(A^{\prime}, X_{0}^{\prime}, G^{\prime}\right) \stackrel{-\otimes_{A^{\prime}} N}{\longrightarrow} \mathcal{M}^{A \otimes k X} \stackrel{\simeq}{\longrightarrow} g r-(A, X, G) .
$$

Then, $N$ is quasi-finite as a right $A \otimes k X$-comodule if and only if $F$ has a left adjoint, if and only if (by Corollary 6.5(2)) there exists an $X \times X_{0}^{\prime}$-graded $A-A^{\prime}$-bimodule $P$ such that $F \simeq \mathrm{H}\left(P_{A^{\prime}},-\right)$, if and only if (by Lemma 6.6, 18, Lemma 3.2(1)] and Theorem [2.3) there exists an $X \times X_{0}^{\prime}$-graded $A-A^{\prime}$-bimodule $P$ such that ${ }_{x} P$ is finitely generated projective in $\mathcal{M}_{A^{\prime}}$ for every $x \in X$, and $N \simeq \mathrm{H}\left(P_{A^{\prime}}, A^{\prime}\right)$ in ${ }^{A^{\prime}} \mathcal{M}^{A \otimes k X}$.

Now let us consider the $X \times X_{0}^{\prime}$-graded $A-A^{\prime}$-bimodule $P:=\mathrm{H}\left({ }_{A^{\prime}} N, A^{\prime}\right)$, and $X^{\prime} \times X$ graded $A^{\prime}-A$-bimodule $M:=\mathrm{H}\left(P_{A^{\prime}}, A^{\prime}\right)$, where $X_{0}^{\prime}$ is a singleton. We have

$$
\begin{gathered}
M=\left\{f \in P^{*} \mid f\left({ }_{x} P\right)=0 \text { for almost all } x \in X\right\} \\
M_{x}=\left\{f \in P^{*} \mid f\left({ }_{y} P\right)=0 \text { for all } y \in X-\{x\}\right\} \quad(x \in X),
\end{gathered}
$$

where $P^{*}=\operatorname{Hom}_{A^{\prime}}\left(P_{A^{\prime}}, A_{A^{\prime}}^{\prime}\right)$. The structure of $A^{\prime}-A$-bimodule on $P^{*}$ is given by $(f a)(p)=$ $f(a p),\left(a^{\prime} f\right)(p)=a^{\prime} f(p)\left(f \in P^{*}, a \in A, a^{\prime} \in A^{\prime}, p \in P\right)$. We have

$$
M \leq\left(P^{*}\right)_{A}, \quad M_{x} \leq M \leq A_{A^{\prime}}\left(P^{*}\right), \quad \text { and } \quad M_{x} \simeq\left({ }_{x} P\right)^{*} \text { in }{ }_{A^{\prime}} \mathcal{M} \quad(x \in X) .
$$

Analogously,

$$
\begin{gathered}
P=\left\{f \in{ }^{*} N \mid f\left(N_{x}\right)=0 \text { for almost all } x \in X\right\} \\
{ }_{x} P=\left\{f \in{ }^{*} N \mid f\left(N_{y}\right)=0 \text { for all } y \in X-\{x\}\right\} \quad(x \in X),
\end{gathered}
$$

where ${ }^{*} N=\operatorname{Hom}_{A^{\prime}}\left({ }_{A^{\prime}} N,{ }_{A^{\prime}} A^{\prime}\right)$. The structure of $A-A^{\prime}$-bimodule on ${ }^{*} N$ is given by $(a f)(n)=f(n a),\left(f a^{\prime}\right)(n)=a^{\prime} f(n)\left(f \in{ }^{*} N, a \in A, a^{\prime} \in A^{\prime}, n \in N\right)$. We have

$$
P \leq{ }_{A}\left({ }^{*} N\right), \quad{ }_{x} P \leq P \leq\left({ }^{*} N\right)_{A^{\prime}}, \quad \text { and } \quad{ }_{x} P \simeq{ }^{*}\left(N_{x}\right) \text { in } \mathcal{M}_{A^{\prime}} \quad(x \in X) .
$$

Hence, $N_{x}$ is finitely generated projective in ${ }_{A^{\prime}} \mathcal{M}$, for every $x \in X$ implies that ${ }_{x} P$ is finitely generated projective in $\mathcal{M}_{A^{\prime}}$, for every $x \in X$, which implies that $M_{x}$ is finitely generated projective in $A^{\prime} \mathcal{M}$, for every $x \in X$.

Finally, let us consider for every $x \in X$ the isomorphism of left $A^{\prime}$-modules

$$
H_{x}: N_{x} \stackrel{\simeq}{\longrightarrow}\left(*\left(N_{x}\right)\right)^{*} \stackrel{\simeq}{\longrightarrow}\left({ }_{x} P\right)^{*} \stackrel{\simeq}{\longrightarrow} M_{x} .
$$

We have $H_{x}\left(n_{x}\right)(\gamma)=\gamma\left(n_{x}\right)$ if $\gamma \in{ }_{x} P$, and $H_{x}\left(n_{x}\right)(\gamma)=0$ if $\gamma \in{ }_{y} P$ and $y \in X-\{x\}$. Set

$$
H=\bigoplus_{x \in X} H_{x}: N \stackrel{\simeq}{\longrightarrow} M .
$$


It can be proved easily that $H$ is a morphism of right $A$-modules. Hence it is an isomorphism of graded bimodules.

(2) We have $\left\{e_{x, i}^{*}, \sigma_{x}\left(e_{x, i}\right)\right\}_{i \in I_{x}}$ is a dual basis of * $\left(N_{x}\right) \simeq{ }_{x} P$, where $\sigma_{x}$ is the evaluation map $(x \in X)$. From the proof of Lemma 6.5 and Remark 6.7, the unit of the adjunction $\left(-\widehat{\otimes}_{A} P,-\otimes_{A^{\prime}} N\right)$ is $\theta_{M}: M \rightarrow M \widehat{\otimes}_{A} P \otimes_{A^{\prime}} N, \theta_{M}(m)=\sum_{x \in X} \sum_{i \in I_{x}} m_{x} \otimes_{A} e_{x, i}^{*} \otimes_{A^{\prime}} e_{x, i}$ $(m \in M)$. By [18, 4.1], the coaction on $\mathrm{h}_{A \otimes k X}(N, M)=M \widehat{\otimes}_{A} P: \rho_{M \widehat{\otimes}_{A} P}: M \widehat{\otimes}_{A} P \rightarrow$ $M \widehat{\otimes}_{A} P \otimes_{A^{\prime}}\left(A^{\prime} \otimes k X^{\prime}\right)$ is the unique $A^{\prime}$-linear map satisfying the commutativity of the diagram

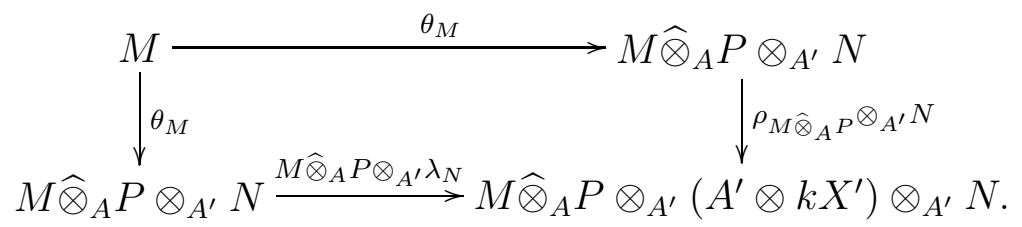

Finally, the commutativity of the above diagram is equivalent to the condition (77).

Now we are in a position to state and prove the main results of this section which characterize adjoint pairs and Frobenius pairs of functors between categories of graded modules over $G$-sets.

Theorem 6.9. Let $M$ be an $X \times X^{\prime}$-graded $A-A^{\prime}$-bimodule, and $N$ be an $X^{\prime} \times X$-graded $A^{\prime}-A$-bimodule. Then the following are equivalent

(1) $\left(-\widehat{\otimes}_{A} M,-\widehat{\otimes}_{A^{\prime}} N\right)$ is an adjoint pair;

(2) $\left(N \widehat{\otimes}_{A^{-}}, M \widehat{\otimes}_{A^{\prime}}\right)$ is an adjoint pair;

(3) $N_{x}$ is finitely generated projective in $A_{A^{\prime}} \mathcal{M}$, for every $x \in X$, and $\widehat{A} \widehat{\otimes}_{A} \mathrm{H}\left({ }_{A^{\prime}} N, A^{\prime}\right) \simeq M$ as $X \times X^{\prime}$-graded $A-A^{\prime}$-bimodules;

(4) there exist bigraded maps

$$
\psi: \widehat{A} \rightarrow M \widehat{\otimes}_{A^{\prime}} N \text { and } \omega: N \widehat{\otimes}_{A} M \rightarrow \widehat{A}^{\prime},
$$

such that

$$
\left(\omega \widehat{\otimes}_{A^{\prime}} N\right) \circ\left(N \widehat{\otimes}_{A} \psi\right)=\Lambda \text { and }\left(M \widehat{\otimes}_{A^{\prime}} \omega\right) \circ\left(\psi \widehat{\otimes}_{A} M\right)=M .
$$

In particular, $\left(-\widehat{\otimes}_{A} M,-\widehat{\otimes}_{A^{\prime}} N\right)$ is a Frobenius pair if and only if $\left(M \widehat{\otimes}_{A^{\prime}}-, N \widehat{\otimes}_{A}-\right)$ is a Frobenius pair.

Proof. We know that the corings $A \otimes k X$ and $A^{\prime} \otimes k X^{\prime}$ are coseparable. The Theorem 2.7 achieves the proof.

Theorem 6.10. Let $G$ and $G^{\prime}$ be two groups, $A$ be a $G$-graded $k$-algebra, $A^{\prime}$ be a $G^{\prime}$-graded $k$-algebra, $X$ be a right $G$-set, and $X^{\prime}$ be a right $G^{\prime}$-set. For a pair of $k$-linear functors $F: g r-(A, X, G) \rightarrow g r-\left(A^{\prime}, X^{\prime}, G^{\prime}\right)$ and $G: g r-\left(A^{\prime}, X^{\prime}, G^{\prime}\right) \rightarrow g r-(A, X, G)$, the following statements are equivalent 
(a) $(F, G)$ is a Frobenius pair;

(b) there exist an $X \times X^{\prime}$-graded $A-A^{\prime}$-bimodule $M$, and an $X^{\prime} \times X$-graded $A^{\prime}-A$-bimodule $N$, with the following properties

(1) ${ }_{x} M$ and $N_{x}$ is finitely generated projective in $\mathcal{M}_{A^{\prime}}$ and ${ }_{A^{\prime}} \mathcal{M}$ respectively, for every $x \in X$, and $M_{x^{\prime}}$ and ${ }_{x^{\prime}} N$ is finitely generated projective in ${ }_{A} \mathcal{M}$ and $\mathcal{M}_{A}$ respectively, for every $x^{\prime} \in X^{\prime}$,

(2) $\widehat{A}^{\prime} \widehat{\otimes}_{A^{\prime}} \mathrm{H}\left({ }_{A} M, A\right) \simeq N$ as $X^{\prime} \times X$-graded $A^{\prime}-A$-bimodules, and $\widehat{A} \widehat{\otimes}_{A} \mathrm{H}\left({ }_{A^{\prime}} N, A^{\prime}\right) \simeq M$ as $X \times X^{\prime}$-graded $A-A^{\prime}$-bimodules,

(3) $F \simeq-\widehat{\otimes}_{A} M$ and $G \simeq-\widehat{\otimes}_{A^{\prime}} N$.

Proof. Straightforward from Theorem 2.11

\subsection{When is the induction functor $T^{*}$ Frobenius?}

Finally, let $f: G \rightarrow G^{\prime}$ be a morphism of groups, $X$ be a right $G$-set, $X^{\prime}$ be a right $G^{\prime}$-set, $\varphi: X \rightarrow X^{\prime}$ be a map such that $\varphi(x g)=\varphi(x) f(g)$ for every $g \in G, x \in X$. Let $A$ be a $G$-graded $k$-algebra, $A^{\prime}$ be a $G^{\prime}$-graded $k$-algebra, and $\alpha: A \rightarrow A^{\prime}$ be a morphism of algebras such that $\alpha\left(A_{g}\right) \subset A_{f(g)}^{\prime}$ for every $g \in G$.

We have, $\gamma: k X \rightarrow k X^{\prime}$ such that $\gamma(x)=\varphi(x)$ for each $x \in X$, is a morphism of coalgebras, and $(\alpha, \gamma):(A, k X, \psi) \rightarrow\left(A^{\prime}, k X^{\prime}, \psi^{\prime}\right)$ is a morphism in $\mathbb{E}_{:}(k)$.

Let $-\otimes_{A} A^{\prime}: g r-(A, X, G) \rightarrow g r-\left(A^{\prime}, X^{\prime}, G^{\prime}\right)$ be the functor making commutative the following diagram

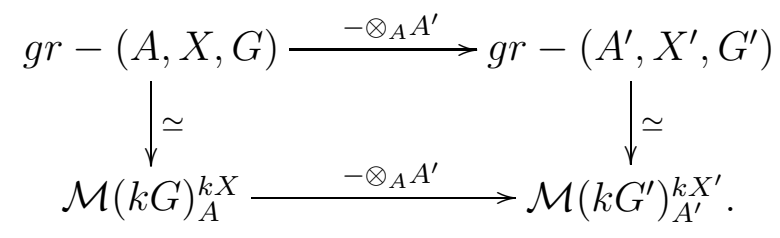

Let $M \in g r-(A, X, G)$. We have $M \otimes_{A} A^{\prime}$ is a right $A^{\prime}$-module, and

$$
\rho^{r}\left(m_{x} \otimes_{A} a_{g^{\prime}}^{\prime}\right)=\left(m_{x} \otimes_{A} a_{g^{\prime}}^{\prime}\right) \otimes \varphi(x) g^{\prime} .
$$

Therefore, $\left(M \otimes_{A} A^{\prime}\right)_{x^{\prime}}$ is the subgroup of $M \otimes_{A} A^{\prime}$ spanned by the elements of the form $m_{x} \otimes_{A} a_{g^{\prime}}^{\prime}$ where $x \in X, g^{\prime} \in G^{\prime}, \varphi(x) g^{\prime}=x^{\prime}, m_{x} \in M_{x}, a_{g^{\prime}}^{\prime} \in A_{g^{\prime}}^{\prime}$, for every $x^{\prime} \in X^{\prime}$.

Therefore, the functor $-\otimes_{A} A^{\prime}: g r-(A, X, G) \rightarrow g r-\left(A^{\prime}, X^{\prime}, G^{\prime}\right)$ is exactly the induction functor $T^{*}$ defined in [25, p. 531]. Hence $T^{*}$ is a Frobenius functor if and only if the induction functor $-\otimes_{A} A^{\prime}: \mathcal{M}^{A \otimes k X} \rightarrow \mathcal{M}^{A^{\prime} \otimes k X^{\prime}}$ is a Frobenius functor (see Section 5). 
Moreover, we have the commutativity of the following diagram

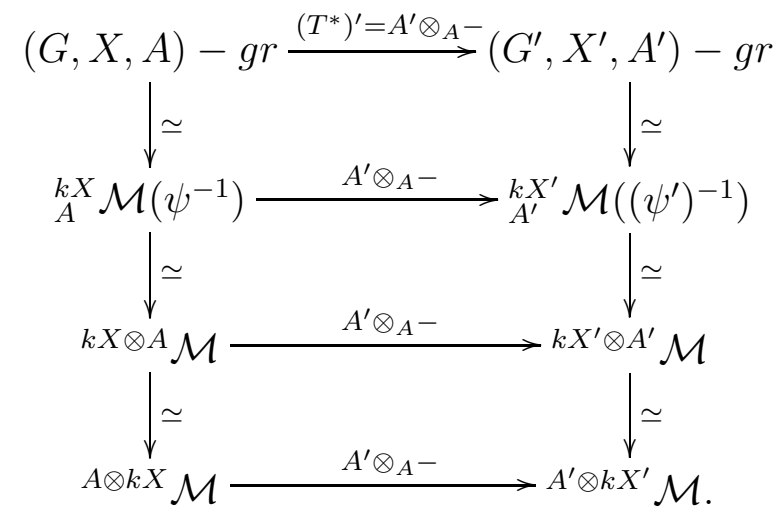

The following consequence of Theorem [5.1] and [25. Theorem 2.27] give two different characterizations when the induction functor $T^{*}$ is a Frobenius functor.

Theorem 6.11. The following statements are equivalent

(a) the functor $T^{*}: g r-(A, X, G) \rightarrow g r-\left(A^{\prime}, X^{\prime}, G^{\prime}\right)$ is a Frobenius functor;

(b) $\left(T^{*}(\widehat{A})\right)_{x^{\prime}}$ is finitely generated projective in ${ }_{A} \mathcal{M}$, for every $x^{\prime} \in X^{\prime}$, and there exists an isomorphism of $X^{\prime} \times X$ graded $A^{\prime}-A$-bimodules

$$
\widehat{A}^{\prime} \widehat{\otimes}_{A^{\prime}} \mathrm{H}\left({ }_{A} T^{*}(\widehat{A}), A\right) \simeq\left(T^{*}\right)^{\prime}(\widehat{A}) .
$$

As an immediate consequence of Proposition 4.6, we obtain

Proposition 6.12. The following are equivalent

(a) the functor $T^{*}: g r-(A, X, G) \rightarrow g r-\left(A^{\prime}, X^{\prime}, G^{\prime}\right)$ is a Frobenius functor;

(b) the functor $\left(T^{*}\right)^{\prime}:(G, X, A)-g r \rightarrow\left(G^{\prime}, X^{\prime}, A^{\prime}\right)-g r$ is a Frobenius functor.

\section{Acknowledgements}

The results in this paper are part of the author's Ph.D. thesis at the University of Granada, Spain. The author would like to thank his advisor, the Professor José Gómez-Torrecillas, for its assistance in the writing of this paper, and for the fruitful discussions.

The author would also like to thank the Professor Edgar Enochs to have communicated to him the example of a commutative self-injective ring which is not coherent. He would like to express his gratitude to the Professor Tomasz Brzeziński for his assistance, for his carefully reading of this paper, and for his useful comments on Section 6. 


\section{References}

[1] K. Al-Takhman, Äquivalenzen zwischen komodul-kategorien von koalgebren über ringen. Ph.D. thesis, Universität Düsseldorf, 1999.

[2] K. Al-Takhman, Equivalences of comodule categories for coalgebras over rings. J. Pure App. Algebra 173 (2002), 245-271.

[3] F.W. Anderson and K.R. Fuller, Rings and categories of modules. Graduate texts in mathematics, 13, Springer Verlag, Second edition, 1992.

[4] T. Brzeziński, Frobenius properties and Maschke-type theorems for entwined modules. Proc. Amer. Math. Soc., 128 (2000), 2261-2270.

[5] T. Brzeziński, The structure of corings: Inductions functors, Maschke type theorem, and Frobenius and Galois-type properties. Algebras and Repr. Theory, 5 (2002), 389410.

[6] T. Brzeziński, Towers of corings. Comm. Algebra, 31 (2003), 2015-2026.

[7] T. Brzeziński and J. Gómez-Torrecillas. On comatrix corings and bimodules. K-Theory, 29 (2003) 101-115.

[8] T. Brzeziński and R. Wisbauer, Corings and comodules. Cambridge University Press, Cambridge, 2003.

[9] S. Caenepeel, E. De Groot and G. Militaru, Frobenius functors of the second kind. Comm. Algebra, 30 (2002), 5357-5389.

[10] S. Caenepeel, E. De Groot, J. Vercruysse, Galois theory for comatrix corings: descent theory, Morita theory, Frobenius and separability properties. Preprint, 2004 (arXiv:math.RA/0406436,v3).

[11] S. Caenepeel, G. Militaru, and S. Zhu, Doi-Hopf modules, Yetter-Drindfel'd modules and Frobenius type properties. Trans. Amer. Math. Soc. 349 (1997), 221-247.

[12] S. Caenepeel, G. Militaru, and S. Zhu, Frobenius and separable functors for generalized module categories and nonlinear equations. Lecture Notes Math. 1787, Springer Verlag, Berlin (2002).

[13] F. Castaño Iglesias, J. Gómez-Torrecillas and C. Năstăsescu, Frobenius functors, Applications. Comm. Algebra, 27 (1999), 4879-4900.

[14] R.R. Colby and K.R. Fuller, Exactness of the double dual and Morita duality for Grothendieck categories. J. Algebra, 82 (1983), 546-558. 
[15] L. El Kaoutit and J. Gómez-Torrecillas, Morita duality for corings over QuasiFrobenius rings. Lecture Notes in Pure and Applied Mathematics (Marcel Dekker), in press.

[16] L. El Kaoutit, J. Gómez-Torrecillas, F. J. Lobillo, Semisimple corings. Algebra Colloquium 11 (2004), 427-442.

[17] P. Freyd, Abelian categories. New York, Harper and Row, 1964.

[18] J. Gómez-Torrecillas, Separable functors in corings. Int. J. Math. Math. Sci. 30 (2002), 203-225.

[19] J. Gómez-Torrecillas and C. Năstăsescu, Quasi-co-Frobenius coalgebras. J. Algebra. 174 (1995), 909-923.

[20] J. Gómez-Torrecillas and C. Năstăsescu, Colby-Fuller duality between coalgebras. J. Algebra 185 (1996), 527-543.

[21] F. Guzman, Cointegrations, relative cohomology for comodules, and coseparable corings. J. Algebra 126 (1989), 211-224.

[22] F. Kasch, Projektive Frobenius-Erweiterungen, Sitzungsber. Heidelberger Akad. Wiss., Math.- Naturw. Kl. 1960/61 (1961), 89?-109.

[23] I.-P. Lin, Semiperfect coalgebras. J. Algebra. 49 (1977), 357-373.

[24] C. Menini, Functors between categories of graded modules. Applications. Bull. Soc. Math. Belg. 45 (1993), 297-315.

[25] C. Menini and C. Năstăsescu, When are induction and coinduction functors are isomorphic?. Bull. Belg. Math. Soc. 1 (1994), 521-558.

[26] B. Mitchell, Theory of categories. Academic Press, New York, 1965.

[27] K. Morita, Adjoint pairs of functors and Frobenius extentions. Sci. Rep. Tokyo Kyoiku Daigaku, Sect. A 9(1965), 40-71.

[28] T. Nakayama and T. Tsuzuku, On Frobenius extensions I. Nagoya Math. J. 17 (1960), 89-110.

[29] C. Năstăsescu, S. Raianu, and F. Van Oystaeyen, Graded modules over G-Sets. Math. Z. 203 (1990), 605-627.

[30] C. Năstăsescu and F. Van Oystaeyen, Methods of graded rings. Lecture Notes Math. 1836, Springer Verlag, Berlin (2004).

[31] N. Popescu, Abelian categories with applications to rings and modules. Academic Press, London, 1973. 
[32] A. Del Río, Categorical methods in graded ring theory. Publ. Math. 36 (1992), 489-531.

[33] B. Stenström, Rings of quotients. Springer, Berlin, 1975.

[34] M. Sweedler, The predual theorem to the Jacobson-Bourbaki theorem. Trans. Amer. Math. Soc. 213 (1975), 391-406.

[35] M. Takeuchi, Formal schemes over fields. Comm. Algebra 5 (1977), 1483-1528.

[36] M. Takeuchi, Morita theorems for categories of comodules. J. Fac. Sci. Univ. Tokyo 24 (1977), 629-644.

[37] M. Zarouali-Darkaoui, Comatrix coring generalized and equivalences of categories of comodules. Preprint 2005.

[38] B. Zimmermann-Huigsen, Pure submodules of direct products of free modules. Math. Ann. 224 (1976), 233-245. 